\title{
GIS - Comprehensive Analytical Approach for Soil Use by Linking Crop Soil Suitability to Soil Management and Reclamation
}

\author{
Abdrabelnabi M. Abd El-Hady ${ }^{1}$ and Emad F. Abdelaty ${ }^{2 *}$
}

\begin{abstract}
Region of Alex-Cairo Desert Road (Egypt) has agricultural potentiality to contribute to food security; therefor the soil of the farm of Nile Company, at $63 \mathrm{Km}$ Alex-Cairo Desert Road, was evaluated by applying our comprehensive analytical approach of evaluation. Soil physical and chemical characterization conducts to soil numerical classification and crops soil suitability that has the advantage to guide the practices of soil management and reclamation.
\end{abstract}

Soil physical characterization leaded to univariate numerical soil classification that pointed that the major phases were moderately soil profile depth (1996.76 Feddan), moderately permeability (3543.90 Feddan), low holding capacity (2608.11 Feddan) and sandy loam textural phases (1800.83 Feddan). Soil chemical characterization led to univariate numerical soil classification which showed that the major classes were moderately saline $\mathbf{( 3 1 2 4 . 7 6}$ Feddan), non-sodic (3531.04 Feddan) and non-calcareous classes (3851.35 Feddan).

The study referred to selection salt tolerant crops as cultural practice for managing soil salinity. Leaching requirements $(\mathrm{LR})$ of reclamation purposes, for different EC-tolerance crops, were determined to output GIS-EC edaphological map. This map that may guide the process of saline soil reclamation was composed of four mapping units having the area of $298.76,3124.76,845.35$ and 16.11 Feddan. The map that determined the spatial distribution of (LR) application showed that: the max allover total leaching water requirements (ATLR) of $13189090.54 \mathrm{~m}^{3}$ are to cultivate all studied area by orange. (b) Wheat is more salts tolerant than orange. Accordingly, the max allover total leach requirements were 1557042.70 $\mathrm{m}^{3}$ /studied soil to plant wheat, which were less greatly than the case of orange cultivation.

GIS-ESP edaphological soil classification was elaborated by assigning ESP thresholds of tolerant crop range to GIS-ESP soil map to produce the GIS-ESP edaphological map. The map divided the studied area into three categories of ESP tolerance crop soil; extremely sensitive ESP crop (1355.09 Feddan), sensitive ESP crop (2845.73 Feddan) and moderately tolerant crop (84.88 Feddan). This edaphological soil classification enabled to calculate edaphological gypsum requirement (GR) for different ESP-tolerance crops. GIS-EC and ESP overlaid maps output the soil multivariable chemical classification. The overlaid GIS-EC-map classified the studied soils into five variants; non saline-non sodic soil (298.80 Feddan) moderately saline-non sodic soil (2701.42 Feddan), highly saline-non sodic soil (535.95 Feddan), moderately salinesodic soil (424.04 Feddan), and highly saline-sodic soil (325.47 Feddan)

Land suitability determined the main limitation factors to guide soil management and reclamation. Wheat soil suitability classified the soils into of the area was conditionally suitable $(\mathrm{S} 4=\mathbf{5 7 . 3 \%})$ and $(\mathrm{S3}=\mathbf{4 2 . 7 \%})$. As for faba bean, the soils had the three classes; marginally suitable $(\mathrm{S3}=\mathbf{4 7 . 6 \%})$, conditionally suitable $(\mathrm{S} 4=30.7 \%)$, and moderately suitable $(\mathrm{S} 2=21.7 \%)$. Grape soil suitability map distributed into two main classes; $63.8 \%$ $($ moderately suitable $=$ S2), $26.1 \%($ marginally suitable $=\mathrm{S3})$. Soils majority was moderately suitable $(\mathrm{S} 2=64.5 \%)$ for olive tree. $61.79 \%($ moderately suitable $=\mathrm{S} 2), \mathbf{1 9 . 6 4} \%$ (marginally suitable $=\mathrm{S3})$, represented the majors of potato soils suitability. The largest area of the study area $(88.02 \%)$ was classified as highly $(\mathrm{S} 1=40.5 \%)$ and moderately suitable $(\mathrm{S} 2=\mathbf{4 7 . 5 \%})$ for tomato.

Keywords: GIS, Soil numerical classification, GIS-EC and ESP overlaid maps, GIS-EC edapholical map, Soil GIS-ESP edapholoical soil classification, Multivariable chemical classification, Soil suitability.

\section{INTRODUCTION}

The economic development of Egypt is highly dependent on agricultural sector that represents around $40 \%$ of the Egyptian workforce and it is the third largest economic sector after tourism and cash remittances from Egyptians working abroad (FAO, 2018a).

Due to wrong and overexploitation of land use in Egypt, the land degradation problem has become an issue of concern. (Abdelaty, 2016) studied an agricultural area (Specific area in Nile Delta) that has been declined by $11.15 \%$ within the period of six years (2008 to 2014) .So, (Mohammed, 2006) considered that the rapidly changing in land use may result a significant resource imbalance. To face this issue, the Egyptian Government has launched the Sustainable Agricultural Development Strategy towards 2030 which aim to reclamation of 1.5 Million Feddan. Goals of this 
Strategy would be achieved when lands were categorized and utilized based upon their different characteristics (Kassim et al., 2018).

Soil numerical classification is an objective classification based on the actual differences between individual soils. The general idea is to minimize withinclass variance, and maximize between-class variance, according to some objective criterion (FAO, 2018b).

Saline and sodic soils are commonly occurring in most part of the world (Prapagar et al., 2012) especially in the arid and semi-arid regions, whereas, globally there are 400 million hectares of land (over 6\% of the world land area) affected by either salinity or sodicity (Gurung and Azad, 2013). Salt-affected soils (saline, sodic and saline-sodic) differ considerably in use suitability, productivity, ease of reclamation, and management (Diaz and Presley, 2017). The leaching has been identified as the most effective method for removal of soluble salts from the rhizosphere in saline soils while application of chemical amendments (such as addition of gypsum) to remove the sodium from the soil's cation exchange sites is necessary to reclaim sodic soils (Prapagar et al., 2012).

Land suitability analysis is a very important technique for agricultural activities to deciding future cropping pattern, planning and activities. It is determining appropriate crops for a specific piece of land according to its characteristics (Singha and Swain, 2016; Pan and Pan, 2012) and allows identification of the limiting factors for the crop cultivation (Joerin et al., 2001).

The coupling of soil suitability model, GIS and remote sensing has become increasingly important for getting the different soil suitability maps that will be guide for decision makers in order to achieve high agricultural productivity (Singha and Swain, 2016).

In Egypt, a number of studies were elaborated to evaluate land suitability for crops (Abdel-Hamid et al., 2010; Aldabaa et al., 2010; Abd-Elmabod et al., 2012; Wahab et al., 2013; ElGhonamey, 2015; Aldabaa and Khralifa, 2016; Afify et al., 2016; ElBaroudy, 2016; Elnaggar, 2017; Fadl and Abuzaid, 2017; Abd El-Aziz, 2018).

The main target aim of this study was to link the GIS- maps of soil numerical classification to soil management and reclamation. While, the other objectives were: (a) precise the practices of soil management and reclamation (b) determine the different soil factors limitation.

\section{MATERIALS AND METHODS}

1. The study area: The study area located in the north eastern part of the Western Desert, it's bounded by the Alexandria Cairo desert road in North Eastern
(785263 E, 3409453 N) and El-Nasr Canal in South Western (776481 E, $3403013 \mathrm{~N})$. It covers about 4285.71 Feddan (Figure 1). El-Nasr canal is the main source of irrigation in the study area.

Agroclimatic data indicated referred that the study area has an aridity index (0.02) to lay in agroclimatic region of hyper-arid, mild winter, warm summer. It has indicated mean annual temperature of $21.2 \mathrm{C}^{\circ}$. The mean monthly temperature ranges from $13.7 \mathrm{C}^{\circ}$ (January) to $27.8 \mathrm{C}^{\circ}$ (August). The highest temperature recorded, as a mean value, was $34.50 \mathrm{C}^{\circ}$ in July and the lowest was $7.80 \mathrm{C}^{\circ}$ (9) January (Abdelaty, 2015).

\section{Elaboration Study}

The study was based on physical and chemical soil characterization that was elaboration through two phases (Figure 2).

\section{Data collection and Georeferenced soil sampling}

Data collection: The data collection including topographic maps and satellite image (ASTER images and Google Earth Pro). Topographic maps were digitized using ArcGIS 10.2 software to produce the base map of GIS soil maps. Satellite image displayed the general location of the studied area (ArcGIS 10.2 software, 2008).

Georeferenced soil sampling: Fifty-one soil samples were collected from twenty-five soil profiles that were located by the global position system (GPS). The GPS was adjusted to acquire UTM coordinates of soil samples (Figure 3). Soil samples were collected depending soil morphological characteristics to represent all soil variations. Twenty five soil profiles were dug to a depth of $120 \mathrm{~cm}$, or to the hard layer, whichever is closer to the soil surface.

\section{Soil Physical and Chemical Characterization}

The collected disturbed soil samples were air-dried, ground gently, and then sieved through $2 \mathrm{~mm}$ sieve to elaborate soil physical and chemical analysis:

Physical analysis: Soil texture determined by hydrometer's method (FAO, 1970), hydraulic conductivity coefficient (K) (Stibinger, 2014) and water holding capacity (Viji and Rajesh, 2012).

Chemical analysis: Electric conductivity (EC), soluble cations ( $\mathrm{Ca}, \mathrm{Mg}$, $\mathrm{Na}$, and $\mathrm{K}$ ), soluble anions $\left(\mathrm{CO}_{3}, \mathrm{HCO}_{3}\right.$, and $\left.\mathrm{Cl}\right)$ of soil past extract, soil $\mathrm{pH}$ of (1:2.5) suspension and calcium carbonate (Page et al., 1982). 


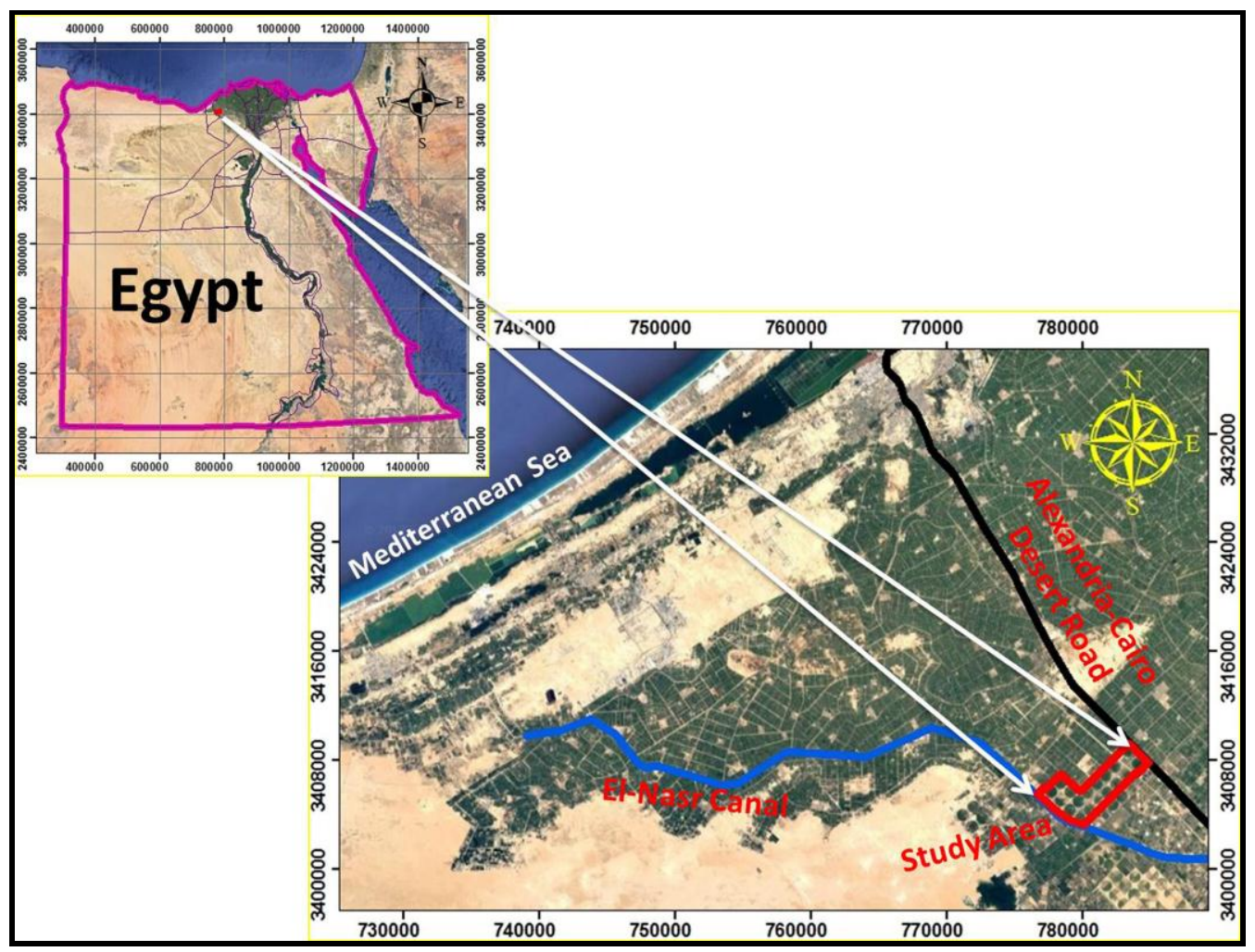

Figure 1. Location of studied area

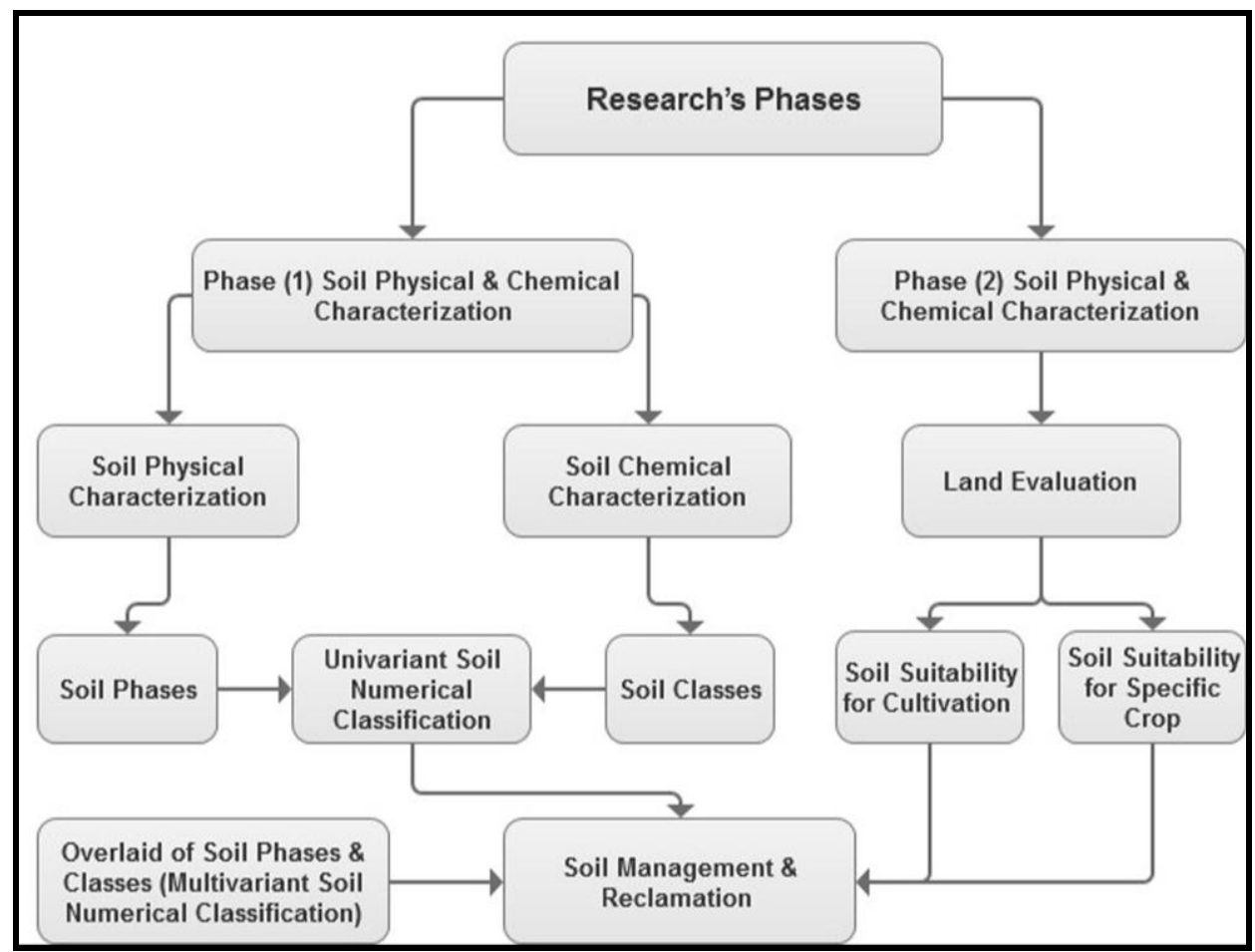

Figure 2. Research flow chart 


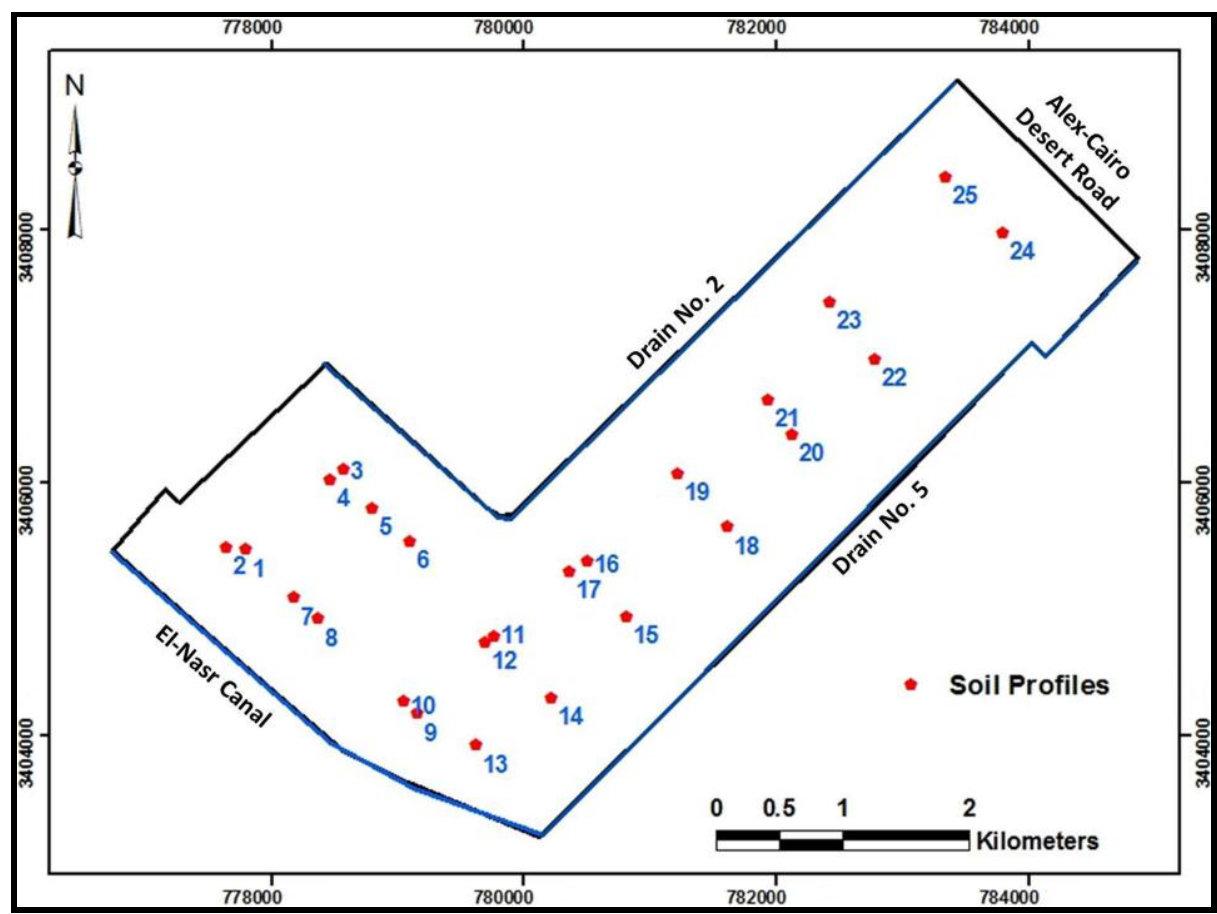

Figure 3. Research flow chart

\section{GIS - data processing of soil chemical and physical}

The data of soil chemical and physical analysis were weighted by arithmetic mean (Mishra, 2004) to be processed to output soil maps (ArcGIS 10.2 software, 2008).

$$
\mathbf{W M}=\frac{\sum_{i=1}^{n} w_{i} x_{i}}{\sum_{i=1}^{n} w_{i}}
$$

Where:

$$
\begin{aligned}
& \mathrm{WM}=\text { Weighted Arithmetic Mean } \\
& \mathrm{Xi}=\text { Variable value (Soil parameter) } \\
& \mathrm{Wi}=\text { weighting factor (Horizon thickness) }
\end{aligned}
$$

Determination of leaching requirements (LR) for different EC-tolerance crops:

The following equation can be used to estimate how much water is required to leach salts for reclamation purposes:

DW $=(K \times$ Ds $\times$ ECei $) /$ ECef, where :

DW $=$ depth of water infiltrated

Ds $\quad=$ soil depth to be reclaimed $=$ crop rooting depth

$\mathrm{K}=0.30$ for fine - textured soils, 0.10 for coarse textured soils
$\mathrm{K}=0.1$ for all soils (sprinkler irrigation and pivot irrigation system)

ECei $=$ initial soil salinity $=$ thresholds of the mapping units or soil salinity classes

Accordingly:

Min. ECein $=$ Min. initial soil salinity $=$ The first (lower) threshold of the mapping unit or soil salinity class (n)

Max . ECein = Max .initial soil salinity $=$ The second (upper) threshold of the mapping unit or soil salinity class (n)

ECef $=$ Desired final soil salinity (target soil salinity $)=$ EC soil paste that enables to obtain zero crop yield (Bauder et al., 2018)

Determination of gypsum requirements (GR) for different ESP-tolerance crops:

ESP values and samples coordinates were input to map GIS-ESP edapoloical soil classification. GIS-ESP map was based on the following information and considerations:

GR (ton gypsum/feddan, by rough method) $=1.7 *$ $\mathrm{KNa}_{\mathrm{ex}}$ *D/30 (Abd El-Hady, 2009), Where;

$\mathrm{D}=$ Crop rooting depth

Naex $=$ exchangeable sodium $(\mathrm{meq} / 100 \mathrm{gm})$

KNaex $=$ Required Naex to be removed from the soil 


\section{Calculate the exchangeable sodium (Naex)}

The studied soils characterized by had moderate cations exchange capacity (CEC) that had value of 25 $\mathrm{meq} / 100 \mathrm{gm}$. The cations exchange capacity was used to calculate the exchangeable sodium (Naex): Naex $(\mathrm{meq} / 100 \mathrm{gm})=\mathrm{ESP} * \mathrm{CEC}=\mathrm{ESP} * 25$

Initial Naex = Initial soil Naex (Naex before gypsum application) ESP

$\mathrm{RNaex}=$ the reference ESP threshold tolerant crop

KNaex = Initial Naex - RNaex

Min. RNaex and Max. RNaex thresholds, of ESP tolerant crop ESP, representing ESP tolerant crop range

Min KNaex = Initial Naex - Min RNaex

Max.KNaex = Initial Naex - Max.RNaex

Application efficiency of gypsum requirements gypsum requirements $(\mathrm{EF})=92 \%$

Gypsum purity (GP) $=93 \% \quad($ Abd El-Hady, 2009)

\section{Land evaluation}

Land was evaluated by applying Agricultural Land Evaluation System for arid region (ALESArid) Model (Abdel Kawy, 2004). ALES-Arid enables to evaluate the suitability for 32 crops to determine the optimum land use. FAO (1979) and Ismail et al., 1994 \& 2001 rated Land suitability classes (Table 1).

Table 1. Land suitability classes

\begin{tabular}{ccc}
\hline Class & Description & Rating (\%) \\
\hline S1 & Highly suitable & $100-80$ \\
S2 & Moderately suitable & $80-60$ \\
S3 & Marginally suitable & $60-40$ \\
S4 & Conditionally suitable & $40-20$ \\
NS1 & Potentially suitable & $20-10$ \\
NS2 & Actually unsuitable & $<10$ \\
\hline
\end{tabular}

\section{RESULTS AND DISCUSSION}

Soil Physical Characterization for Univariate Soil Numerical Classification

Soil physical characterization: Table (2) summarizes some soil physical characteristics; profile depth, hydraulic conductivity (Ks), water holding capacity (WHC) and soil texture. The minimal soil profile depth was $43 \mathrm{~cm}$ (profile 24), and maximal was $120 \mathrm{~cm}$. The min. value of soil hydraulic conductivity was $4.3 \mathrm{~cm} / \mathrm{hr}$ in subsurface sample (profile 14), and max. value reached to $12 \mathrm{~cm} / \mathrm{hr}$ samples; $1,15,17,20,23$ and 25 . Water holding capacity ranged between the minimum value $10 \%$ (surface samples of profiles, 11 and 15), and maximum value $38 \%$ (subsurface sample, profile 2 ). The soil texture for the study area varied from sandy clay loam (sample 48) to sand textured sample (21).

Soil Physical-Univariate Numerical Classification for Soil Management and Reclamation: Soil profile depth, soil texture, water holding capacity, and hydraulic conductivity (Ks) were separately used to classify the studied soil into different phases. A soil phase is a unit of soil outside the system of soil taxonomy. It is a functional unit that may be designed according to the purpose of the survey (Canada Government, 1998). Meanwhile, the term of soil class was restricted to denote soil mapping units that are chemically different.

- Soil Profile Depth: Most of the soil profiles were characterized by hard layer; therefore the classification of the profile depth was based on it (depth and hardness). Soil profiles were classified into phases; deep soil $(>95 \mathrm{~cm})$, moderately deep soil $(70-95 \mathrm{~cm})$ and shallow soil $(<70 \mathrm{~cm})$ that were presented by area of 1632.42, 1996.76 and 656.78 Feddan, respectively (Table 3 and Figure 4).

- Soil Texture: Mechanical analysis results showed that the studied soil were grouped four into textural phases; sand (312.00 Feddan), sandy clay Loam (583.54 Feddan), loamy sand (1589.35 Feddan) and sandy loam (1800.83 Feddan) (Table 3 and Figure 4).

- Hydraulic Conductivity (Ks): Soil hydraulic conductivity was determined due to the importance of soil permeability in the soil drainage condition and the growth of crops. The hydraulic conductivity values categorized the soil area into three categories; slow permeability (163.33 Feddan), moderately permeability (3543.90 Feddan) and rapid permeability (577.76 Feddan) with Ks values of $<7.5,7.5-10$ and $>10 \mathrm{~cm} / \mathrm{hr}$ (Table 3 and Figure 4). The moderately permeability phase (3543.90 Feddan) dominated the area with 82.70 $\%$.

- Water Holding Capacity (WHC): The studied soil was characterized by the dominance of the phase of low field water holding capacity $(<15 \%)$ that represented an area of 2608.11 Feddan. $(60.87 \%)$. The minor area $(12.31 \%)$ represented the high WHC soil phase $(>20 \%)$ (Table 3 and Figure 4).

Soil Management and Reclamation (Basing on Univariant Soil Physical Numerical Classification):

Managing hard layered-soil: The rooting depth may be affected by the soil depth, constraining soil layers (hardpan, plow pan, etc.), or even abrupt changes in soil texture, Division of Agriculture and Natural Resources, University of California (2018). Soil profile depth, plants rooting and soil penetration resistance determined the cultivated crops selection. The soil penetration resistance of 150 psi that corresponds to $50 \%$ root penetration (Duiker, 2002) was assigned as a threshold to classify studied soils into phases of none-little and 
slight-moderate penetration resistance. The research formulated the cultivation crops selection as a function of soil profile depth and plants rooting (Table 4).

- Reclamation of hard layered-soil: Generally, hard layered-soil can be reclaimed by deep tillage and sulfur and organic fertilizers applications. Lowering soil $\mathrm{pH}$ conducts to dissolve calcium carbonate that cements the soil particles forming the hard pan (Abd El-Hady, 2009).

Soil Chemical Characterization for Univariate and multivariante Soil Numerical Classification

Soil Chemical Characterization: Table (5) summarizes some soil chemical characteristics; pH, EC,
ESP and $\mathrm{CaCO}_{3}$. $\mathrm{pH}$ values indicated that soils were alkaline (7.48) to extremely alkaline (8.70). EC had a wide range to be between the minimum value of 0.05 $\mathrm{ds} / \mathrm{m}$ (subsurface horizon, profile 15$)$ and $(19.84 \mathrm{ds} / \mathrm{m})$ as maximal (surface horizon, profile 10). Soils classes varied from nonsodic class having ESP of $2.71 \%$ (surface horizon, profile 17) to extremely sodic class that characterized by maximal ESP of $27.38 \%$ (surface horizon, profile 13). $\mathrm{CaCO}_{3}$ ranged between the minimum value $(4.93 \%)$ in subsurface sample of profile number 22 and 25 to the maximum value $(40.36 \%)$ in subsurface sample of profile number 19.

Table 2. Main soil physical characteristics

\begin{tabular}{|c|c|c|c|c|c|c|c|c|c|c|c|}
\hline \multicolumn{2}{|c|}{ Profile } & \multirow{2}{*}{$\begin{array}{l}\text { Sample N } \\
\text { (Depth, } \\
\text { cm) }\end{array}$} & \multirow[b]{2}{*}{$\begin{array}{c}\mathrm{Ks} \\
(\mathrm{cm} / \mathrm{hr})\end{array}$} & \multirow[b]{2}{*}{$\begin{array}{c}\text { WHC } \\
(\%)\end{array}$} & \multirow{2}{*}{$\begin{array}{c}\text { Textural } \\
\text { Class } \\
\end{array}$} & \multicolumn{2}{|c|}{ Profile } & \multirow[b]{2}{*}{$\begin{array}{l}\text { Sample N } \\
\text { (Depth, cm) }\end{array}$} & \multirow[b]{2}{*}{$\begin{array}{c}\mathrm{Ks} \\
(\mathrm{cm} / \mathrm{hr})\end{array}$} & \multirow[b]{2}{*}{$\begin{array}{c}\text { WHC } \\
(\%)\end{array}$} & \multirow{2}{*}{$\begin{array}{c}\text { Textural } \\
\text { Class } \\
\end{array}$} \\
\hline $\mathrm{N}$ & $\frac{\text { Depth }}{(\mathrm{cm})}$ & & & & & $\mathrm{N}$ & $\frac{\text { Depth }}{(\mathrm{cm})}$ & & & & \\
\hline \multirow{2}{*}{1} & \multirow{2}{*}{120} & $1(0-35)$ & 11 & 15 & $\mathrm{LS}$ & \multirow{3}{*}{14} & \multirow{3}{*}{88} & $27(0-30)$ & 7 & 13 & $\mathrm{~S}$ \\
\hline & & $2(35-120)$ & 12 & 15 & LS & & & $28(30-52)$ & 6.5 & 12 & $\mathrm{~S}$ \\
\hline \multirow{2}{*}{2} & \multirow{2}{*}{85} & $3(0-41)$ & 5 & 22 & LS & & & $29(52-88)$ & 4.3 & 15 & SL \\
\hline & & $4(41-85)$ & 6 & 38 & SCL & \multirow{2}{*}{15} & \multirow{2}{*}{88} & $30(0-30)$ & 12 & 10 & S \\
\hline \multirow{2}{*}{3} & \multirow{2}{*}{120} & $5(0-37)$ & 8.5 & 16 & SCL & & & $31(30-88)$ & 11 & 12 & $\mathrm{~S}$ \\
\hline & & $6(37-120)$ & 8 & 18 & SCL & \multirow{2}{*}{16} & \multirow{2}{*}{120} & $32(0-58)$ & 7 & 13 & LS \\
\hline \multirow{2}{*}{4} & \multirow{2}{*}{95} & $7(0-44)$ & 8.5 & 16 & LS & & & $33(58-120)$ & 7 & 15 & SL \\
\hline & & $8(44-95)$ & 8 & 14 & SCL & \multirow{2}{*}{17} & \multirow{2}{*}{120} & $34(0-55)$ & 11 & 14 & $\mathrm{~S}$ \\
\hline \multirow{3}{*}{5} & \multirow{2}{*}{120} & $9(0-38)$ & 8 & 13 & SL & & & $35(55-120)$ & 12 & 18 & SL \\
\hline & & $10(38-120)$ & 9 & 12 & SCL & \multirow{2}{*}{18} & 00 & $36(0-33)$ & 6.5 & 18 & LS \\
\hline & & $11(0-28)$ & 8.5 & 16 & LS & & 90 & $37(33-90)$ & 6 & 17 & SCL \\
\hline 0 & 120 & $12(28-120)$ & 9 & 18 & LS & 10 & 120 & $38(0-55)$ & 8.5 & 13 & $\mathrm{LS}$ \\
\hline & 85 & $13(0-33)$ & 8 & 13 & S & 19 & 120 & $39(55-120)$ & 8 & 14 & SL \\
\hline I & 85 & $14(33-85)$ & 8.5 & 13 & SCL & 20 & 00 & $40(0-45)$ & 11 & 16 & SCL \\
\hline & & $15(0-28)$ & 8 & 20 & SL & 20 & 90 & $41(45-90)$ & 12 & 13 & SCL \\
\hline 8 & 92 & $16(28-92)$ & 7.5 & 14 & SCL & 21 & 88 & $42(0-38)$ & 8 & 13 & LS \\
\hline 9 & 66 & $17(0-36)$ & 7.5 & 16 & SL & 21 & 88 & $43(38-88)$ & 8.5 & 22 & S \\
\hline 9 & 60 & $18(36-66)$ & 8 & 13 & LS & & & $44(0-55)$ & 7 & 18 & SCL \\
\hline 10 & 65 & $19(0-30)$ & 8 & 13 & $S$ & 22 & 83 & $45(55-83)$ & 6.5 & 23 & LS \\
\hline 10 & ט & $20(30-65)$ & 8.5 & 13 & SL & 23 & 83 & $46(0-55)$ & 12 & 20 & SL \\
\hline 11 & 120 & $21(0-55)$ & 9.5 & 10 & S & 23 & 83 & $47(55-83)$ & 11 & 13 & S \\
\hline 11 & 120 & $22(55-120)$ & 9 & 15 & SL & & & $48(0-25)$ & 9 & 18 & LS \\
\hline & & $23(0-50)$ & 8.5 & 17 & S & 24 & 43 & $49(25-43)$ & 9.5 & 22 & SL \\
\hline 12 & 120 & $24(50-120)$ & 9.5 & 11 & $S$ & & & $50(0-38)$ & 11 & 17 & LS \\
\hline & & $25(0-28)$ & 7.5 & 18 & SCL & 25 & 88 & $51(38-88)$ & 12 & 23 & LS \\
\hline 13 & 88 & $26(28-88)$ & 8.5 & 24 & SCL & $\begin{array}{l}*: \mathrm{L} \\
\mathrm{S}=\mathrm{S}\end{array}$ & $\begin{array}{l}\mathrm{s}=\text { Loamy } \\
\text { and }\end{array}$ & Sand, $\mathrm{SCL}=\mathrm{Sar}$ & dy Clay I & $\mathrm{m}, \mathrm{SL}=$ & andy Loam, \\
\hline
\end{tabular}




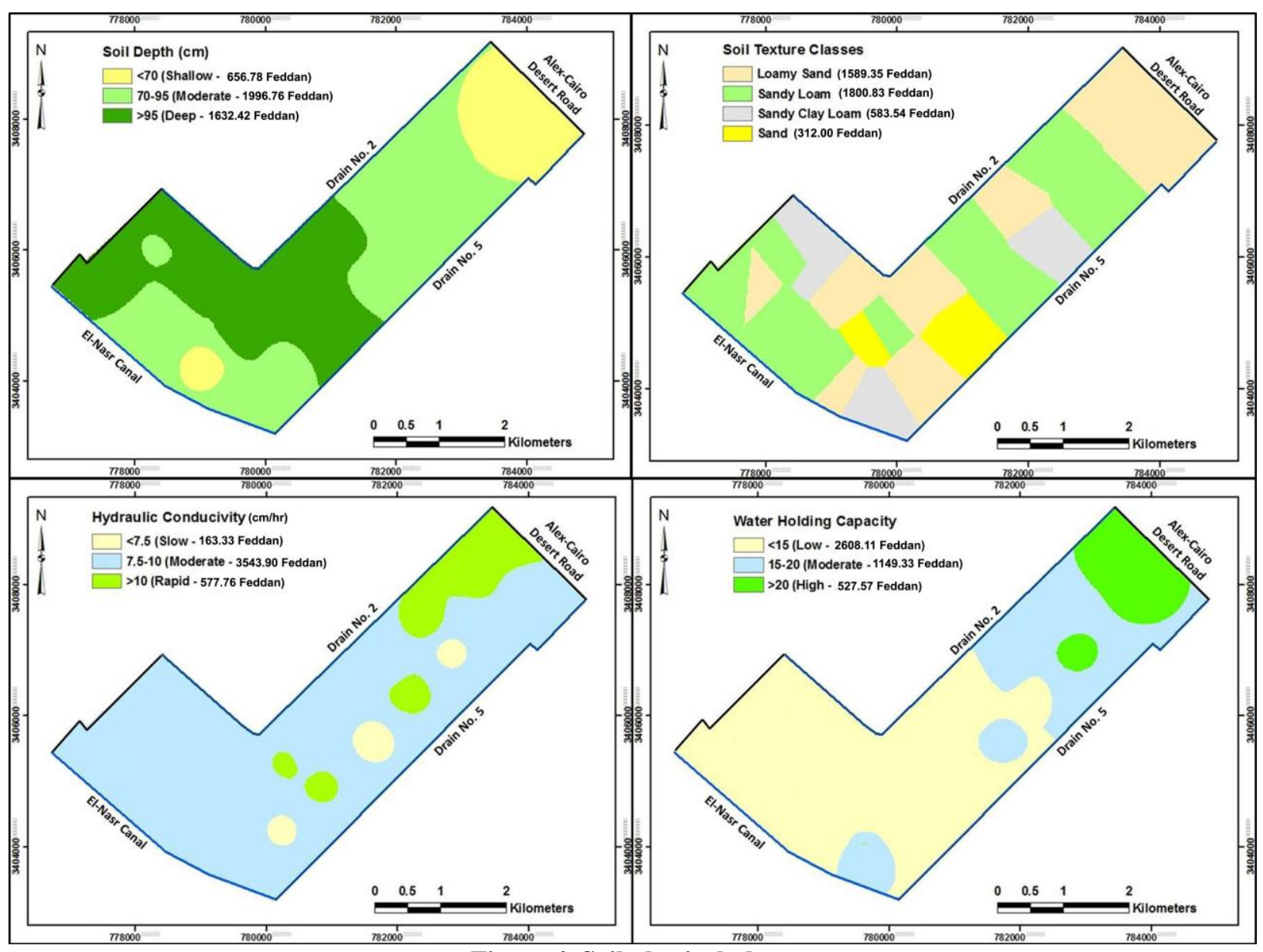

Figure 4. Soil physical phases

Table 3. Univariant soil physical classification

\begin{tabular}{|c|c|c|c|c|c|c|c|c|c|}
\hline \multirow{2}{*}{$\begin{array}{c}\text { Soil } \\
\text { Physical } \\
\text { Criteria }\end{array}$} & \multirow[b]{2}{*}{ Thresholds } & \multirow{2}{*}{$\begin{array}{l}\text { Soil } \\
\text { Phases }\end{array}$} & \multicolumn{2}{|c|}{ Area } & \multirow{2}{*}{$\begin{array}{c}\text { Soil } \\
\text { Physical } \\
\text { Criteria }\end{array}$} & \multirow[b]{2}{*}{ Thresholds } & \multirow[b]{2}{*}{$\begin{array}{c}\text { Soil } \\
\text { Phases }\end{array}$} & \multicolumn{2}{|c|}{ Area } \\
\hline & & & Feddan & $\%$ & & & & Feddan & $\%$ \\
\hline \multirow{4}{*}{ Depth $\mathrm{cm}$} & $<70$ & Shallow & 656.78 & 15.32 & Hydraulic & $<7.5$ & Slow & 163.33 & 3.81 \\
\hline & $70-95$ & Moderate & 1996.76 & 46.59 & Conductivity & $7.5-10$ & Moderate & 3543.90 & 82.70 \\
\hline & $>95$ & Deep & 1632.42 & 38.09 & $(\mathrm{Ks}) \mathrm{cm} / \mathrm{hr}$ & $>10$ & Rapid & 577.76 & 13.48 \\
\hline & & LS & 1589.35 & 37.08 & Water & $<15$ & Low & 2608.11 & 60.87 \\
\hline \multirow{3}{*}{ Texture } & & SCL & 583.54 & 13.62 & Holding & $15-20$ & Moderate & 1149.33 & 26.82 \\
\hline & $\begin{array}{l}\text { Textural } \\
\text { Classes }\end{array}$ & SL & 1800.83 & 42.02 & $\begin{array}{l}\text { Capacity } \\
(\text { WHC) }(\%)\end{array}$ & $>20$ & High & 527.57 & 12.31 \\
\hline & & $\mathrm{S}$ & 312.00 & 7.28 & & & & & \\
\hline
\end{tabular}


Table 4. Crop selection as function of soil profile depth, plants rooting and soil penetration resistance

\begin{tabular}{|c|c|c|c|c|c|c|c|}
\hline \multicolumn{2}{|c|}{ Soil profile depth } & \multicolumn{2}{|c|}{ Soil Penetration Resistance } & \multirow{2}{*}{$\begin{array}{c}\text { Recommended } \\
\text { Sub soiling } \\
\text { Tillage } \\
\end{array}$} & \multirow[b]{2}{*}{$\begin{array}{l}\text { Recommended } \\
\text { Crops }\end{array}$} & \multicolumn{2}{|c|}{ Area } \\
\hline $\begin{array}{l}\text { Thresholds } \\
\text { (cm) }\end{array}$ & $\begin{array}{c}\text { Soil } \\
\text { Phases }\end{array}$ & $\begin{array}{c}\text { Thresholds } \\
\text { (psi) }\end{array}$ & Soil Phases & & & Feddan & $\%$ \\
\hline$<70$ & $\begin{array}{l}\text { Shallow } \\
\text { soil } \\
\text { profile }\end{array}$ & $<150$ & $\begin{array}{c}\text { None - Little } \\
\text { penetration } \\
\text { resistance }\end{array}$ & No & $\begin{array}{c}\text { garlic } \\
\text {,cucumber } \\
\text { spinach ,onion } \\
\text {, lettuce }\end{array}$ & 656.78 & 15.32 \\
\hline $70-95$ & $\begin{array}{l}\text { Moderate } \\
\text { depth-soil } \\
\text { profile }\end{array}$ & $>150$ & $\begin{array}{l}\text { Slight - } \\
\text { Moderate } \\
\text { penetration } \\
\text { resistance }\end{array}$ & Yes & $\begin{array}{l}\text { carrot, beets, } \\
\text { Potatoes, beans }\end{array}$ & 1996.76 & 46.59 \\
\hline $\begin{array}{c}>95 \\
150 \mathrm{psi}=10\end{array}$ & $\begin{array}{l}\text { Deep soil } \\
\text { profile } \\
\text { ar according }\end{array}$ & the manual & & & All crops & 1632.42 & 38.09 \\
\hline
\end{tabular}

Table 5. Main soil chemical characteristics

\begin{tabular}{|c|c|c|c|c|c|c|c|c|c|c|c|}
\hline $\begin{array}{c}\text { Profile } \\
\text { N. }\end{array}$ & $\frac{\text { Sample }}{\text { N. }}$ & pH & $\begin{array}{c}\text { EC } \\
d s / m\end{array}$ & ESP & $\frac{\mathrm{CaCO}_{3}}{(\%)}$ & $\frac{\text { Profile }}{\text { N. }}$ & $\frac{\text { Sample }}{\text { N. }}$ & pH & $\begin{array}{c}\mathrm{EC} \\
\mathrm{ds} / \mathrm{m}\end{array}$ & ESP & $\frac{\mathrm{CaCO}_{3}}{(\%)}$ \\
\hline \multirow{2}{*}{1} & 1 & 7.6 & 10.85 & 8.09 & 10.76 & \multirow{3}{*}{14} & 27 & 8.16 & 4.49 & 16.65 & 13 \\
\hline & 2 & 7.98 & 4.02 & 4.11 & 8.97 & & 28 & 8.38 & 2.27 & 8.54 & 8.52 \\
\hline \multirow{2}{*}{2} & 3 & 7.6 & 10.85 & 8.09 & 10.76 & & 29 & 8.06 & 3.79 & 4.42 & 16.59 \\
\hline & 4 & 8.21 & 5.78 & 11.98 & 9.87 & \multirow[b]{2}{*}{15} & 30 & 7.73 & 6.22 & 6.26 & 14.35 \\
\hline \multirow{2}{*}{3} & 5 & 8.07 & 4.42 & 7.92 & 21.52 & & 31 & 8.26 & 0.05 & 6.75 & 14.35 \\
\hline & 6 & 8.23 & 1.06 & 6.14 & 21.52 & \multirow{2}{*}{16} & 32 & 7.75 & 6.96 & 10.3 & 8.52 \\
\hline \multirow[b]{2}{*}{4} & 7 & 8.17 & 1.17 & 6.49 & 7.17 & & 33 & 7.87 & 3.28 & 9.52 & 12.11 \\
\hline & 8 & 8.26 & 1.06 & 12.74 & 21.52 & \multirow{2}{*}{17} & 34 & 8.03 & 0.57 & 2.71 & 9.42 \\
\hline \multirow{2}{*}{5} & 9 & 7.69 & 8.23 & 6.15 & 12.11 & & 35 & 7.87 & 11.53 & 10.41 & 23.32 \\
\hline & 10 & 7.86 & 2.35 & 10.8 & 23.77 & \multirow[b]{2}{*}{18} & 36 & 7.61 & 4.93 & 15.71 & 13.45 \\
\hline \multirow{2}{*}{6} & 11 & 7.48 & 7.51 & 16.24 & 7.62 & & 37 & 8.04 & 3.39 & 12.51 & 11.66 \\
\hline & 12 & 7.65 & 13.41 & 21 & 12.56 & \multirow{2}{*}{19} & 38 & 7.48 & 7.51 & 16.24 & 7.62 \\
\hline \multirow{2}{*}{7} & 13 & 8.15 & 4.96 & 12.76 & 7.17 & & 39 & 8.7 & 1.64 & 13.48 & 40.36 \\
\hline & 14 & 8.04 & 7.04 & 22.68 & 20.18 & \multirow{2}{*}{20} & 40 & 7.79 & 4.99 & 11.01 & 17.49 \\
\hline \multirow{2}{*}{8} & 15 & 8.33 & 1.35 & 6.01 & 23.32 & & 41 & 8.1 & 3.45 & 9.25 & 26.46 \\
\hline & 16 & 8.35 & 0.78 & 12 & 17.04 & \multirow{2}{*}{21} & 42 & 7.74 & 9.83 & 5.76 & 14.35 \\
\hline \multirow{2}{*}{9} & 17 & 8.1 & 13.12 & 12.76 & 14.35 & & 43 & 7.62 & 11.53 & 8.13 & 12.11 \\
\hline & 18 & 7.85 & 5.9 & 18.28 & 8.52 & \multirow[b]{2}{*}{22} & 44 & 7.88 & 2.03 & 6.41 & 8.52 \\
\hline \multirow{2}{*}{10} & 19 & 7.7 & 19.84 & 15.52 & 22.87 & & 45 & 8.68 & 0.09 & 3.66 & 4.93 \\
\hline & 20 & 7.76 & 14.55 & 12.74 & 26.01 & \multirow{2}{*}{23} & 46 & 8.08 & 4.9 & 24 & 16.59 \\
\hline \multirow{2}{*}{11} & 21 & 8.04 & 9.52 & 9.21 & 10.31 & & 47 & 7.98 & 3.71 & 12.91 & 13.9 \\
\hline & 22 & 8.26 & 1.06 & 9.92 & 19.73 & \multirow{2}{*}{24} & 48 & 7.65 & 13.41 & 21 & 12.56 \\
\hline \multirow{2}{*}{12} & 23 & 7.82 & 4.25 & 6.39 & 7.17 & & 49 & 8.6 & 4.75 & 26.23 & 21.52 \\
\hline & 24 & 7.82 & 8.74 & 8.65 & 17.94 & \multirow{3}{*}{25} & 50 & 8.19 & 0.33 & 5.01 & 6.73 \\
\hline \multirow{2}{*}{13} & 25 & 8.08 & 6.47 & 27.38 & 13.9 & & 51 & 8.68 & 0.09 & 3.66 & 4.93 \\
\hline & 26 & 8.22 & 3.11 & 22.67 & 7.17 & & & & & & \\
\hline
\end{tabular}

Soil Univariant Chemical Classification for Management and Reclamation:

The studied soils were chemically classified basing separately on the thresholds of $\mathrm{pH}, \mathrm{EC}, \mathrm{ESP}$ and $\mathrm{CaCO}_{3}$ that are the most effective factors on soil production:
- Soil Salinity: The results of electrical conductivity indicated that the studied soils were generally positioned into the moderately saline class that had an area of (3124.76 Feddan) representing (72.92 \%). The soils assembled into four classes; low saline (298.76 Feddan) and it is suitable for most crops, moderately saline (3124.76 Feddan), high saline (845.35 Feddan) where 
crops salt tolerant crops give yield with marginal reduction. Finally, very high saline class (16.11 Feddan) that it is suitable just for high salt tolerant crops but also with yield reduction (Table 6 and Figure 5).

- Soil Sodicity: Results showed that the non-sodic soil class occupied the majority of the studied area with $82.40 \%$ (3531.04 Feddan). The sodic soil had only 753.95 Feddan (17.60 \%) (Table 6 and Figure 5).

- Calcium Carbonate Content: Calcium carbonate of the study area had different forms such as powder, nodules, concretions and hard layer. Calcium carbonate in calcareous soil gives it one of the important morphological phenomena, which is the surface crust. The study area was classified into three classes according to percentage of calcium carbonate to: non calcareous soil (3851.35 Feddan), moderately calcareous soil (411.85 Feddan) and calcareous soil (21.78 Feddan) (Table 6 and Figure 5).

Soil Management and Reclamation (Basing on soil chemical univariate classification):

\section{Soil Salinity Problem}

- Managing Soil Salinity: Suitable crops were selected to reduce the problem of soil salinity (Table 7).

- Reclamation of saline soil by determination of leaching requirements (LR) for different ECtolerance crops: The equation of Bauder et al., 2018 was applied to estimate how much water is required to leach salts for reclamation purposes. Crops tolerance and EC soil parameters that are EC tolerant crop range and soil initial $\mathrm{EC}\left(\mathrm{EC}_{\mathrm{ei}}\right)$ were calculated and showed in table 7.These parameters were assigned to the equation, Bauder et al. (2018), to calculate the depth of water infiltrated (DW) (Table 8). DW was used to determine the min and max allover net LR (ANLR), and min and max allover total LR (ATLR), tables $(9,10)$. The tables pointed to the following outputs:

- To cultivate orange in the mapping units $(1,2,3,4)$ the total leaching water requirements (TLR), of 98414.5 (min), $393661.1(\max ), 4117308.8(\min ), 8234648.8$ (max), $2227742.4(\min ), 4455493.3(\max )$, and 84909.2 (min), $105287.4(\max ), \mathrm{m}^{3}$ water/mapping unit, are needed to be applied respectively. This briefly means that max allover total leaching water requirements (ATLR) of $13189090.54 \mathrm{~m}^{3}$ are to cultivate all studied by orange (Table 9 ).

- Wheat is more salts tolerant that orange, whereas ECef has the values of 6.0 and $1.7(\mathrm{dS} / \mathrm{m})$, for wheat and orange, respectively. In addition the rooting depth of wheat and orange are 50 and $120 \mathrm{~cm}$. Accordingly, the max allover total leach requirements were 1557042.70 m3/studied soil (Table 10), to plant wheat, which are less than the case of orange cultivation.

- $296.47 \mathrm{~m}^{3}$ water were needed as minimum net leaching requirements to reclaim soil feddan (NLRF), of mapping unit (1), to be cultivated by orange. This value certainly is not logically acceptable. Min ECei representing the first threshold mapping unit (1) is less than ECef for the case of orange cultivation. Min ECei equals $1(\mathrm{ds} / \mathrm{m})$, while ECef for the case of orange cultivation has the value of $1.7(\mathrm{ds} / \mathrm{m})$. Thus, there no need to min NLRF application. This same false value was found in the case of wheat cultivation, where min NLRF equaled to $35 \mathrm{~m}^{3}$ water. This conclude that The leaching requirements (LR) equation of Bauder et al., 2018, is not applicable when min ECei (initial EC soil ) is lower than ECef (EC reference crop) because it outputs false values indicating the LR needs.

Table 6. Univariant soil chemical classification

\begin{tabular}{|c|c|c|c|c|c|c|c|c|c|}
\hline \multirow{2}{*}{$\begin{array}{c}\text { Soil } \\
\text { chemical } \\
\text { Criteria } \\
\end{array}$} & \multirow[b]{2}{*}{ Thresholds } & \multirow{2}{*}{$\begin{array}{l}\text { Soil } \\
\text { Chemical } \\
\text { Classes }\end{array}$} & \multicolumn{2}{|c|}{ Area } & \multirow{2}{*}{$\begin{array}{c}\text { Soil } \\
\text { chemical } \\
\text { Criteria }\end{array}$} & \multirow[b]{2}{*}{ Thresholds } & \multirow{2}{*}{$\begin{array}{l}\text { Soil } \\
\text { Chemical } \\
\text { Classes }\end{array}$} & \multicolumn{2}{|c|}{ Area } \\
\hline & & & Feddan & $\%$ & & & & Feddan & $\%$ \\
\hline $\begin{array}{l}\text { Exchangeable } \\
\text { Sodium }\end{array}$ & $<15$ & Non-Sodic & 3531.04 & 82.40 & & $<4$ & Low & 298.76 & 6.97 \\
\hline \multirow[t]{2}{*}{$\begin{array}{l}\text { Percentage } \\
\text { (ESP) } \%\end{array}$} & $>15$ & Sodic & 753.97 & 17.60 & Electrical & $4-8$ & Moderate & 3124.76 & 72.92 \\
\hline & $<15$ & $\begin{array}{l}\text { Non } \\
\text { Calcareous }\end{array}$ & 3851.35 & 89.88 & $\begin{array}{l}\text { Conductivity } \\
\text { (EC) ds/m }\end{array}$ & $8-16$ & High & 845.35 & 19.73 \\
\hline \multirow[t]{2}{*}{$\mathrm{CaCO} 3 \%$} & $15-20$ & $\begin{array}{l}\text { Moderately } \\
\text { Calcareous }\end{array}$ & 411.85 & 9.61 & & $>16$ & $\begin{array}{l}\text { Very } \\
\text { High }\end{array}$ & 16.11 & 0.38 \\
\hline & $>20$ & Calcareous & 21.80 & 0.51 & & & & & \\
\hline
\end{tabular}




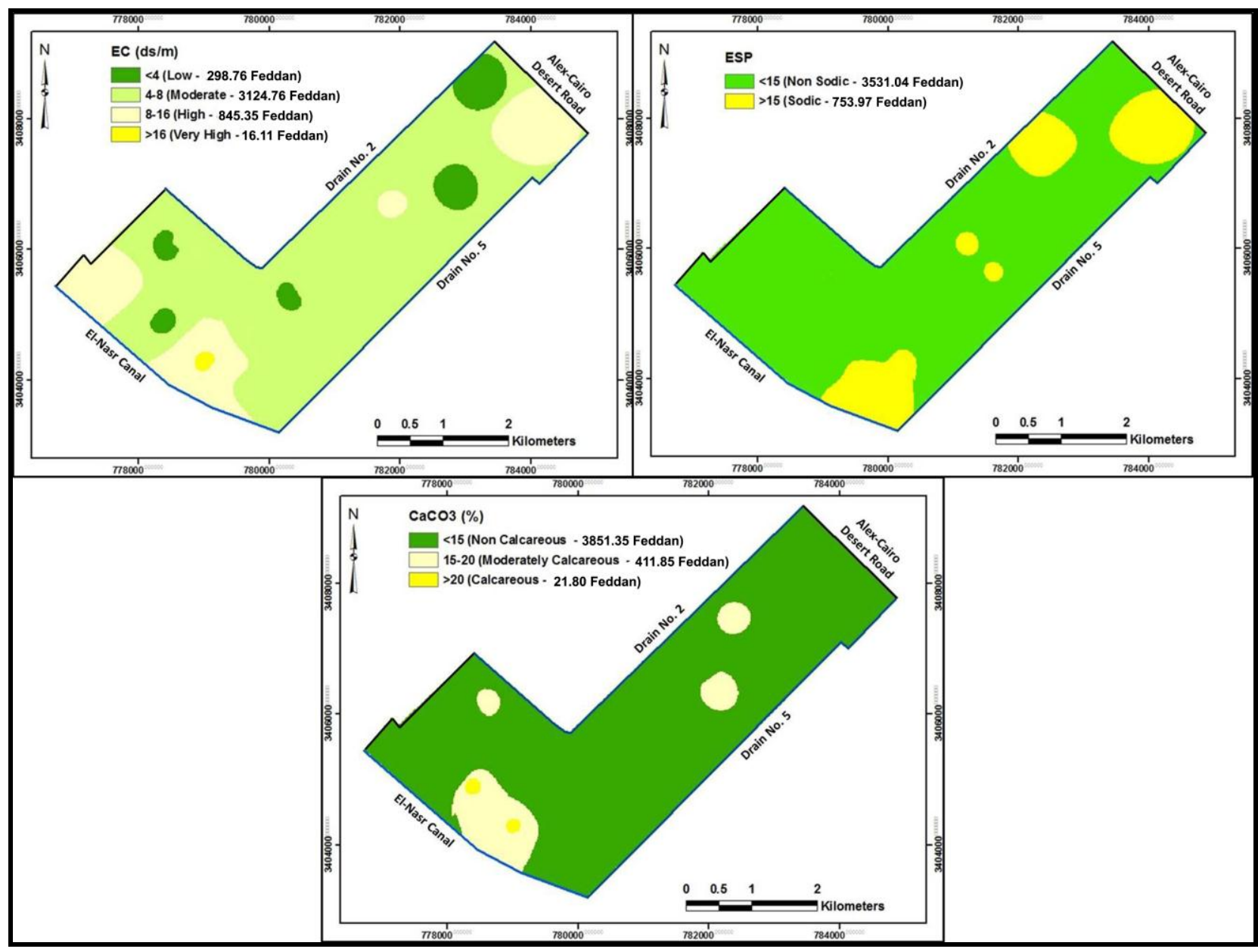

Figure 5. Soil chemical classes

Table 7. Crops tolerance and EC soil parameters

\begin{tabular}{|c|c|c|c|c|}
\hline \multirow{2}{*}{$\begin{array}{l}\text { Mapping } \\
\text { Unit }\end{array}$} & \multirow{2}{*}{ EC Crop Tolerance } & \multirow{2}{*}{$\begin{array}{c}\text { EC } \\
(\mathrm{dS} / \mathrm{m}) \\
\text { tolerant } \\
\text { crop } \\
\text { range } \\
\end{array}$} & \multicolumn{2}{|c|}{$\begin{array}{l}\text { Initial EC } \text { ei }_{\text {(dS/m) }} \\
\quad\end{array}$} \\
\hline & & & Min. & Max. \\
\hline 1 & $\begin{array}{l}\text { Sensitive EC crops: (Field crops) Sunflower, Soybean, Faba bean, } \\
\text { Lins, (Vegetable crops) Sweet corn, Lettuce, Onion, Eggplant, } \\
\text { Carrot, (Fruit crops) Date, Olive, Peach, Orange, Grapes }\end{array}$ & $1-4$ & 1 & 4 \\
\hline 2 & $\begin{array}{l}\text { Moderately EC tolerant crops: only field crops Barley, Cotton, } \\
\text { Sugar beet, Grain sorghum, Wheat }\end{array}$ & $4-8$ & 4 & 8 \\
\hline 3 & EC tolerant crops: No crops have $0 \%$ yield reduction & $8-16$ & 8 & 16 \\
\hline 4 & Highly EC tolerant crops: No crops have $0 \%$ yield reduction & $16-32$ & 16 & 32 \\
\hline
\end{tabular}


Table 8. Depth of water infiltrated (DW) $\operatorname{ECei}(\mathbf{d S} / \mathbf{m})$

\begin{tabular}{cccccc} 
& & \multicolumn{2}{c}{ Orange } & \multicolumn{2}{c}{ Wheat } \\
\cline { 2 - 6 } & & DW $(\mathbf{c m})$ & MW $(\mathbf{c m})$ \\
\hline Min & Max & Min & Max & Min & Max \\
1 & 4 & 7.06 & 28.24 & 0.83 & 3.33 \\
4 & 8 & 28.24 & 56.47 & 3.33 & 6.66 \\
8 & 16 & 56.47 & 112.94 & 6.66 & 13.33 \\
16 & 19.84 & 112.94 & 140.05 & 13.33 & 16.53 \\
\hline
\end{tabular}

$\overline{\mathrm{K}}($ constant $)=0.10$, Ds (rooting depth $)=120$ and $50 \mathrm{~cm}$ for orange and wheat ECef $(\mathrm{EC}$ soil paste that enables to obtain zero orange yield $)=1.7$ and $6(\mathrm{dS} / \mathrm{m})$ for orange and wheat $\left(\mathrm{Max} \mathrm{EC}_{\mathrm{ei}}=\max\right.$. initial $\mathrm{EC}$ of the studied soils $19.84(\mathrm{dS} / \mathrm{m})$

Table 9. Leaching requirements (LR) to reclaim soil to cultivate orange

\begin{tabular}{|c|c|c|c|c|c|c|c|c|c|}
\hline \multirow{3}{*}{$\begin{array}{c}\text { Mapping } \\
\text { Unit }\end{array}$} & \multicolumn{4}{|c|}{ NLR \& TLR $\left(\mathrm{m}^{3}\right.$ water/feddan $)$} & \multirow{3}{*}{$\begin{array}{c}\text { Area } \\
\text { (feddan) }\end{array}$} & \multicolumn{4}{|c|}{ NLRU \& TLRU ( m $^{3}$ water / mapping unit) } \\
\hline & \multicolumn{2}{|c|}{ N LRF } & \multicolumn{2}{|c|}{ TLRF } & & \multicolumn{2}{|c|}{ NLRU } & \multicolumn{2}{|c|}{ TLRU } \\
\hline & Min. & Max. & Min. & Max. & & Min. & Max. & Min. & Max. \\
\hline 1 & 296.47 & 1185.88 & 329.41 & 1317.65 & 298.76 & 88573.38 & 354293.5 & 98414.5 & 393661.1 \\
\hline 2 & 1185.88 & 2371.76 & 1317.64 & 2635.29 & 3124.76 & 3705590.39 & 7411180.8 & 4117308.8 & 8234648.8 \\
\hline 3 & 2371.76 & 4743.53 & 2635.29 & 5270.59 & 845.35 & 2004967.32 & 4009943.1 & 2227742.4 & 4455493.3 \\
\hline 4 & 4743.53 & 5881.98 & 5270.59 & 6535.53 & 16.11 & 76418.27 & 94758.7 & 84909.2 & 105287.4 \\
\hline $\begin{array}{l}\text { All studied } \\
\text { Soils } \\
\text { The leaching } \\
\text { values indic } \\
\text { NLRF and T } \\
\text { NLRU and } \\
\text { ANLR and } \\
\text { Application }\end{array}$ & $\begin{array}{l}\text { Min. allov } \\
\text { Max. allov } \\
\text { Min. allov } \\
\text { Max. allov } \\
\text { equirements } \\
\text { ng the LR n } \\
\text { RF = Net an } \\
\text { RU = Net a } \\
\text { LR = Net a } \\
\text { ficiency of } 1\end{array}$ & $\begin{array}{l}\text { net LR (AI } \\
\text { net LR (AI } \\
\text { total LR GF } \\
\text { total LR (A } \\
\text { LR) equatio } \\
\text { ds. That is } \\
\text { total water } \\
\text { total water } \\
\text { total water } \\
\text { ching (LF) }\end{array}$ & $\begin{array}{l}\text { LR) }= \\
\text { R) = } \\
(\text { ATLR) = } \\
\text { LR) = } \\
\text { of Bauder } \\
\text { t true } \\
\text { aching req } \\
\text { eaching rec } \\
\text { eaching rec } \\
90 \%\end{array}$ & $\begin{array}{l}5875549.35 \\
1870176.07 \\
28374.90 \\
3189090.54 \\
\text { al., 2018, is } \\
\text { ements, for } \\
\text { rements, for } \\
\text { rements, for }\end{array}$ & $\begin{array}{l}\text { 3/ studied so } \\
\mathrm{n}^{3} / \mathrm{studied} \mathrm{s} \\
\text { studied soil } \\
\mathrm{m}^{3} / \mathrm{studied} \mathrm{s} \\
\text { applicable } \\
\text { amation pur } \\
\text { lamation pu } \\
\text { lamation pu }\end{array}$ & $\begin{array}{l}\text { l) min ECei is } \\
\text { ses, for feddan } \\
\text { oses, for mappir } \\
\text { oses, for all map }\end{array}$ & $\begin{array}{l}\text { lower than } \mathrm{F} \\
\left(\mathrm{m}^{3} \text { water/fe }\right. \\
\text { ng unit }\left(\mathrm{m}^{3} \mathrm{v}\right. \\
\text { pping units, }\end{array}$ & $\begin{array}{l}\text { f because it } \\
\text { er) } \\
\text { / mapping } \\
\text { water / all s }\end{array}$ & $\begin{array}{l}\text { puts false } \\
\text { it) } \\
\text { ied soils) }\end{array}$ \\
\hline
\end{tabular}

Table 10. Leaching requirements (LR) to reclaim soil to cultivate wheat

\begin{tabular}{|c|c|c|c|c|c|c|c|c|c|}
\hline \multirow{3}{*}{$\begin{array}{l}\text { Mapping } \\
\text { Unit }\end{array}$} & \multicolumn{4}{|c|}{ NLR \& TLR (m3/feddan) } & \multirow{3}{*}{$\begin{array}{c}\text { Area } \\
\text { (feddan) }\end{array}$} & \multicolumn{4}{|c|}{ NLRU \& TLRU (m3/ mapping unit) } \\
\hline & \multicolumn{2}{|c|}{ N LRF } & \multicolumn{2}{|c|}{ TLRF } & & \multicolumn{2}{|c|}{ NLRU } & \multicolumn{2}{|c|}{ TLRU } \\
\hline & Min. & Max. & Min. & Max. & & Min. & Max. & Min. & Max. \\
\hline 1 & 35 & 140 & 38.89 & 155.56 & 298.76 & 10456.6 & 41826.4 & 11618.8 & 46475.1 \\
\hline 2 & 140 & 280 & 155.56 & 311.11 & 3124.76 & 437466.4 & 874932.8 & 486087.7 & 972144.1 \\
\hline 3 & 280 & 560 & 311.11 & 622.22 & 845.35 & 236698.0 & 473396.0 & 262996.8 & 525993.7 \\
\hline \multirow[t]{2}{*}{4} & 560 & 694.4 & 622.22 & 771.56 & 16.11 & 9021.6 & 11186.8 & 10023.9 & 12429.8 \\
\hline & \multicolumn{5}{|c|}{ Min. allover net LR (AN LR) } & tudied soil) & & & \\
\hline \multirow{3}{*}{$\begin{array}{l}\text { All studied } \\
\text { Soils }\end{array}$} & \multicolumn{5}{|c|}{ Max. allover net LR (AN LR) } & studied soil) & & & \\
\hline & \multicolumn{9}{|c|}{ Min. allover total LR GR (ATLR) = $770727.24(\mathrm{~m} 3 /$ studied soil $)$} \\
\hline & \multicolumn{5}{|c|}{ Max. allover total LR (ATLR) } & studied soil) & & & \\
\hline
\end{tabular}

The leaching requirements (LR) equation of Bauder et al., 2018, is not applicable when min ECei is lower than ECef because it outputs false values indicating the $\mathrm{LR}$ needs. That is not true

Wheat reference $\mathrm{EC}=\mathrm{ECef}=6.0(\mathrm{dS} / \mathrm{m})=\mathrm{EC}$ soil paste that enables to obtain zero wheat yield

Application efficiency of leaching (LF) $=90 \%$

\section{Soil Sodicity Problem}

- Managing Sodic Soil by application of Cultural methods: Applying friendly ESP irrigation and optimal selection of ESP crops tolerant and friendly ESP irrigation water conduct to reliable soil sodicity management.

- For the studied soils they are irrigated by Nile water that has values of EC $(0.51 \mathrm{ds} / \mathrm{m})$ and SAR (1.76)
(Abdelaty, 2018). These low values classified Nile water as suitable irrigation water, even for sensitive crops (Lenntech, 2018). While, Kupper, (1996) considered that there values may cause a moderate risk for sensitive crops. Irrigation water can be practiced in the light of EC-SAR standard tables of Kupper, 1996 and Lenntech, 2018.

- The standard tables of ESP tolerance crops (Ggeorge, 1960; Qadir, et al., 2008) enabled to determine the more 
suitable crops to be cultivated in the studied soils (Table 11). The table indicated that extremely sensitive ESP crops, Nuts, Citrus and Avocado, can only be cultivated in the class of non sodic soil $(\mathrm{ESP}=2-10 \%)$. The sensitive ESP crops, such as beans, may be planted with some yield reduction in the soil having ESP range of 1020. Meanwhile, non sodic soils can be cultivated different crops having, ESP wide range, from sensitive ESP crops to friendly ESP.

- Reclamation of Sodic Soil by determination of edaphological soil gypsum requirements for different ESP-tolerance crops: The determination of edaphological soil gypsum requirements (GR) was based on classification ESP-tolerance crops (Qadir et al., 2008) and $\mathrm{Na}_{\mathrm{ex}}$ soil parameters. GIS-ESP edapholoical soil classification was elaborated by assignment ESP thresholds of tolerant crop range (Table 12) to GIS-ESP soil map (Figure 6). The GIS-ESP edapholoical soil map divided the studied area into three categories of ESP tolerance crops soil; extremely sensitive ESP crop (1355.09 Feddan), sensitive ESP crop (2845.73 Feddan) and moderately tolerant crop (84.88 Feddan). The calculation of edaphological gypsum requirement (GR) for different ESP-tolerance crops (Tables 13, 14 and 15) indicated that:

- Mapping unit (1) can be used to cultivate all the crops, even sensitive ones (such as citrus), without any gypsum application. The case of cultivation mapping unit (2 and 3 ) by such crops, required gypsum applications of 417115.1 and 53221.73 tons as $\min$ and max allover net GR (ANGR), respectively. Consequently amounts of 463461.2 and 59135.26 tons gypsum must be applied such as min and max allover actual GR (ATGR), respectively (Table 13-b).

- Sensitive ESP crops such as beans can be cultivated in the mapping units (1) and (2) without any gypsum application. Nevertheless, is preferable to notify that the second mapping unit may needs to GR in the future because RNaex $=$ Soil initial Naex. This application is necessary to avoid any probable $\mathrm{Na}$ effect on the crop. As for mapping unit (3), it may be suitable soils for planting by cultivate sensitive ESP crops by applying GR of 360.78 and 721.57 tons as min and max NGRU, and 400.87 and 801.74 tons as min and max TGRU. Contrary, gypsum application will not be need in the case of planting tolerant and most crops (Table 14-b).

- There is no need for GR to cultivate moderately tolerant ESP crops in all studied soils because RNaex $\geq$ Soil initial Naex.

- GIS-ESP map enabled to assess not only the gypsum requirements (GR) but also their optimum spatial distribution.
Managing $\mathrm{CaCO}_{3} \%$ problem: Alkalinity that was due calcium carbonate represented a minor problem, where alkaline soils occupied only $10.11 \%$ of the studied area. Chicken manure application of $400 \mathrm{~kg} / \mathrm{fed}(950 \mathrm{~kg} / \mathrm{ha})$ reduced greatly the alkalinity problem to increase plant micronutrients uptake (Wafaa, 2013).

This information and GIS area of moderately calcareous and calcareous soil phases (433.66 Feddan) enabled to not only calculate chicken manure application (173.03 ton) but also determined the application locations by map of figure 5.

\section{Soil Chemical Multivariate Classification:}

The overlay process output the soil multivariable chemical classification maps, as resulted from overlay two characteristics by EC and ESP as shown in figure 7. This map involved five classes. The largest class was of moderately saline-non sodic soil class which represents about $63 \%$ total study area. Non saline-non sodic soil was the smallest one to represent about $7 \%$ total study area.

\section{Land evaluation:}

Soil Suitability for Cultivation: The Agricultural Land Evaluation System for Arid Region program (ALES-Arid) was used to predict soil suitability for some common crops cultivated in the study area including: wheat, faba_bean, grape, olive, potato and tomato. Table (16) summarizes agriculture soil suitability for each profile for the selected crops. This table represents a guide of soil reclamation that can be carried out by removal of restricted factors.

The six suitability classes for each crop are the following: Class S1: Highly suitable, Class S2: Moderately suitable, Class S3: Marginally suitable, Class S4: Conditionally suitable, NS1: Potentially suitable and NS2: Actually unsuitable.

\section{Crops Soil suitability:}

- Cereals crops (Exa. Wheat): The data indicated that the studied soil is marginally suitable and conditionally suitable for wheat crop. The class of conditionally suitable (S4) and marginally suitable soils (S3) represent about $57.28 \%$ and $42.72 \%$ of the studied area, respectively (Table 17 and Figure 8). This low degree of soil suitability may due to the coarse texture and high soil permeability.

- Leguminous crops (Exa. Feba-bean): The data indicated that the studied soil is moderately suitable, marginally suitable and conditionally suitable for Faba bean crop.

The class of marginally suitable soils (S3) represents about $47.65 \%$, conditionally suitable class (S4) represents about $30.65 \%$ and moderately suitable soils 
(S2) represents about $21.70 \%$ of the studied area (Table 17 and Figure 8).

- Perennial crops (Exa. Grape and Olive): Mostly, the grapes can grow in wide range of soil types. According to Bucelli, and Costantini, 2009, the ideal soil for grapes is a well-drained sandy loam soil. Soil $\mathrm{pH}$ of 5.5 to 8 is an ideal to grow grapes, because at this level, nutrients are available for the root (Lanyon et al., 2004). The results showed that all studied area was suitable for grape plantation. The largest portion of the study area $(63.81 \%)$ was classified as moderately suitable (S2). The remaining portions $(26.08 \%, 6.89 \%$ and $3.22 \%$ ) were classified as marginally suitable (S3), highly suitable (S1) and conditionally suitable (S4), respectively (Table 17 and Figure 8).

In order to define soil suitability for the growth of olive trees a number of criteria concerning to the soil properties were delineated. These properties were all

\begin{tabular}{|c|c|c|c|c|}
\hline \multirow{2}{*}{$\begin{array}{c}\text { Soil } \\
\text { Chemical } \\
\text { Classes } \\
\end{array}$} & \multirow{2}{*}{$\begin{array}{c}\text { ESP } \\
\text { Thresholds }\end{array}$} & \multicolumn{2}{|c|}{ Area } & \multirow[b]{2}{*}{ ESP Crops Tolerant } \\
\hline & & Feddan & $\%$ & \\
\hline Non-Sodic & $<15$ & 3531.04 & 82.40 & $\begin{array}{l}\text { Extremely sensitive ESP Crops( ESP = 2-10) ; Nuts , Citrus, } \\
\text { Avocado }\end{array}$ \\
\hline Sodic & $>15$ & 753.97 & 17.60 & $\begin{array}{l}\text { Sensitive ESP crops }(\mathrm{ESP}=10-20) \text {; Beans } \\
\text { Moderately tolerant }(\mathrm{ESP}=20-40) ; \text { Clover } \\
\text { Tolerant crops }(\mathrm{ESP}=(40-60) ; \text { Wheat, Cotton, Alfalfa } \\
\text { Barely, Tomato, Beets } \\
\text { Most tolerant crops ( ESP }>60) ; \text { Tall wheat grass, Rhodes } \\
\text { grass }\end{array}$ \\
\hline
\end{tabular}

Table 12. ESP crops tolerance and Naex soil parameters

\begin{tabular}{clccccc}
\hline \multirow{2}{*}{$\begin{array}{c}\text { Mapping } \\
\text { Unit }\end{array}$} & \multicolumn{1}{c}{ Crop Tolerance } & ESP & \multicolumn{2}{c}{ ESP Thresholds } & \multicolumn{2}{c}{ Initial Naex } \\
\cline { 6 - 7 } & Tolerant & Crop Range & Min. & Max. & Min. & Max. \\
\hline 1 & Extremely sensitive ESP crops: citrus & $2-10$ & 2 & 10 & 0.5 & 2.5 \\
2 & Sensitive ESP crops : beans & $10-20$ & 10 & 20 & 2.5 & 5 \\
3 & Moderately tolerant crops : clover & $20-40$ & 20 & 40 & 5 & 10 \\
\hline
\end{tabular}

Table 13- a. Thresholds of extremely sensitive ESP crops and Naex parameters

\begin{tabular}{ccccccccc}
\hline Mapping Unit & $\begin{array}{c}\text { Thresholds of ESP } \\
\text { crops Tolerance }\end{array}$ & \multicolumn{2}{c}{ Initial Naex } & \multicolumn{2}{c}{ RNaex } & \multicolumn{2}{c}{ KNaex } \\
\hline & Min & Max & Min & Max & Min & Max & Min & Max \\
1 & 2 & 10 & 0.5 & 2.5 & 0.5 & 2.5 & 0 & 0 \\
2 & 10 & 20 & 2.5 & 5 & 0.5 & 2.5 & 2 & 2.5 \\
3 & 20 & 40 & 5 & 10 & 0.5 & 2.5 & 4.5 & 7.5 \\
\hline
\end{tabular}


Table 13-b. Gypsum requirement (GR) to reclaim soil to cultivate extremely sensitive ESP crops Citrus = rooting depth (D) $120 \mathrm{~cm}$

\begin{tabular}{|c|c|c|c|c|c|c|}
\hline \multirow{2}{*}{ Mapping Unit } & \multicolumn{2}{|c|}{ Area } & \multicolumn{2}{|c|}{ NGRU } & \multicolumn{2}{|c|}{ TGRU } \\
\hline & feddan & $\%$ & Min & Max & Min & Max \\
\hline 1 & 1355.66 & 31.40 & \multicolumn{4}{|c|}{ No Need for Gypsum Application* } \\
\hline 2 & 2876.02 & 66.63 & 391138.7 & 48892.34 & 43459.86 & 54324.82 \\
\hline 3 & 84.89 & 1.97 & 2597.63 & 4329.39 & 2886.26 & 4810.43 \\
\hline \multirow{4}{*}{ All studied Soils } & \multicolumn{5}{|c|}{ Min allover net GR (ANGR), (ton/ studied soil) } & 417115.1 \\
\hline & \multicolumn{5}{|c|}{ Max allover net GR (ANGR), (ton/ studied soil) } & 53221.73 \\
\hline & \multicolumn{5}{|c|}{ Min allover total GR (ATGR), (ton/ studied soil) } & 463461.2 \\
\hline & \multicolumn{5}{|c|}{ Max allover total GR (ATGR), (ton/ studied soil) } & 59135.26 \\
\hline
\end{tabular}

$\mathrm{EF}=$ efficiency of gypsum application $=95 \%, \mathrm{GP}=$ gypsum purity $=95 \%$

Allover total gypsum requirements $($ ATGR $)=($ ANGR $) \times 100 / 90 \quad($ ton/ studied soil $)$

*No need for Gypsum Application when RNaex $\geq$ Soil initial Naex

Table 14- a. Thresholds of sensitive ESP crops and Naex parameters

\begin{tabular}{cccccccccc}
\hline \multirow{2}{*}{ Mapping Unit } & $\begin{array}{c}\text { Thresholds of } \\
\text { ESP crops } \\
\text { Tolerance }\end{array}$ & Initial Naex & RNaex & \multicolumn{2}{c}{ KNaex } \\
\cline { 2 - 10 } & Min & Max & Min & Max & Min & Max & Min & Max \\
\hline 1 & 2 & 10 & 0.5 & 2.5 & 0.5 & 2.5 & -2 & -2.5 \\
2 & 10 & 20 & 2.5 & 5 & 0.5 & 2.5 & 0 & 0 \\
3 & 20 & 40 & 5 & 10 & 0.5 & 2.5 & 2.5 & 5 \\
\hline
\end{tabular}

Table 14-b. Gypsum requirement (GR) to reclaim soil to cultivate sensitive ESP crops Beans = rooting depth (D) $=30 \mathrm{~cm}$

\begin{tabular}{|c|c|c|c|c|c|c|}
\hline \multirow{2}{*}{ Mapping Unit } & \multicolumn{2}{|c|}{ Area } & \multicolumn{2}{|c|}{ NGRU } & \multicolumn{2}{|c|}{ TGRU } \\
\hline & feddan & $\%$ & Min & Max & Min & Max \\
\hline 1 & 1355.66 & 31.40 & \multicolumn{4}{|c|}{ No Need for Gypsum Application* } \\
\hline 2 & 2876.02 & 66.63 & \multicolumn{4}{|c|}{ No Need for Gypsum Application* } \\
\hline 3 & 84.89 & 1.97 & 360.78 & 721.57 & 400.87 & 801.74 \\
\hline \multicolumn{6}{|c|}{ Min allover net GR (ANGR), (ton/ studied soil) } & 360.78 \\
\hline \multirow{3}{*}{ All studied Soils } & \multicolumn{5}{|c|}{ Max allover net GR (ANGR), (ton/ studied soil) } & 721.57 \\
\hline & \multicolumn{5}{|c|}{ Min allover total GR (ATGR), (ton/ studied soil) } & 400.87 \\
\hline & \multicolumn{5}{|c|}{ Max allover total GR (ATGR), (ton/ studied soil) } & 801.74 \\
\hline
\end{tabular}

$\mathrm{EF}=$ efficiency of gypsum application $=95 \%, \mathrm{GP}=$ gypsum purity $=95 \%$

Allover total gypsum requirements $($ ATGR $)=($ ANGR $) \times 100 / 90 \quad($ ton/ studied soil $)$

*No need for Gypsum Application when RNaex $\geq$ Soil initial Naex

Table 15. Thresholds of to cultivate moderately tolerant ESP crops and Naex parameters

\begin{tabular}{cccccccccc}
\hline \multirow{2}{*}{ Mapping Unit } & $\begin{array}{l}\text { Thresholds of } \\
\text { ESP crops } \\
\text { Tolerance }\end{array}$ & Initial Naex & RNaex & \multicolumn{2}{c}{ KNaex } \\
\cline { 2 - 10 } & Min & Max & Min & Max & Min & Max & Min & Max \\
\hline 1 & 2 & 10 & 0.5 & 2.5 & 5 & 10 & -4.5 & -7.5 \\
2 & 10 & 20 & 2.5 & 5 & 5 & 10 & -2.5 & -5 \\
3 & 20 & 40 & 5 & 10 & 5 & 10 & 0 & 0 \\
\hline
\end{tabular}

*No need for Gypsum Application when RNaex $\geq$ Soil initial Naex

Clover $=$ rooting depth $(D)=30 \mathrm{~cm}$ 


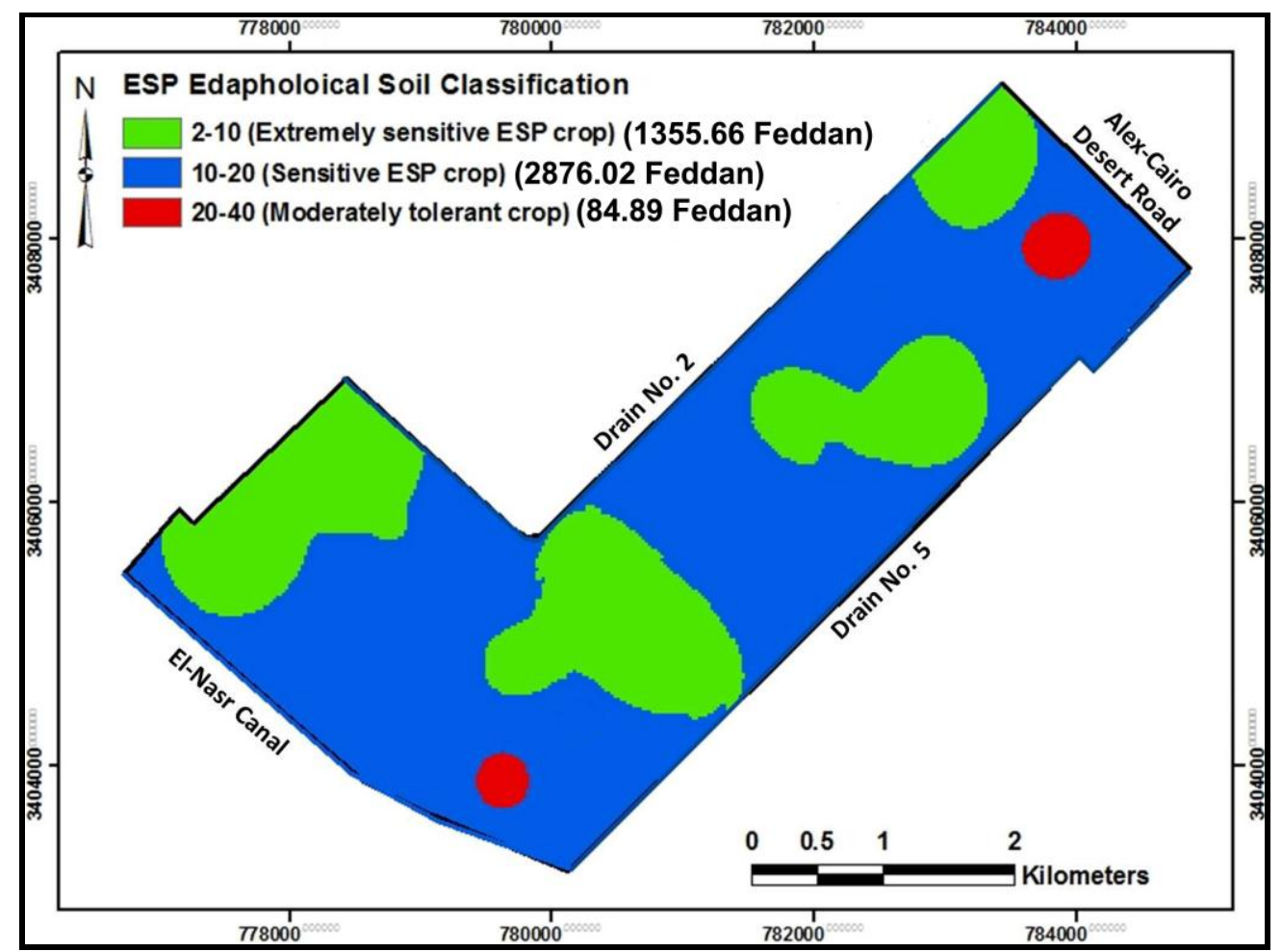

Figure 6. GIS-ESP edapholoical soil classification

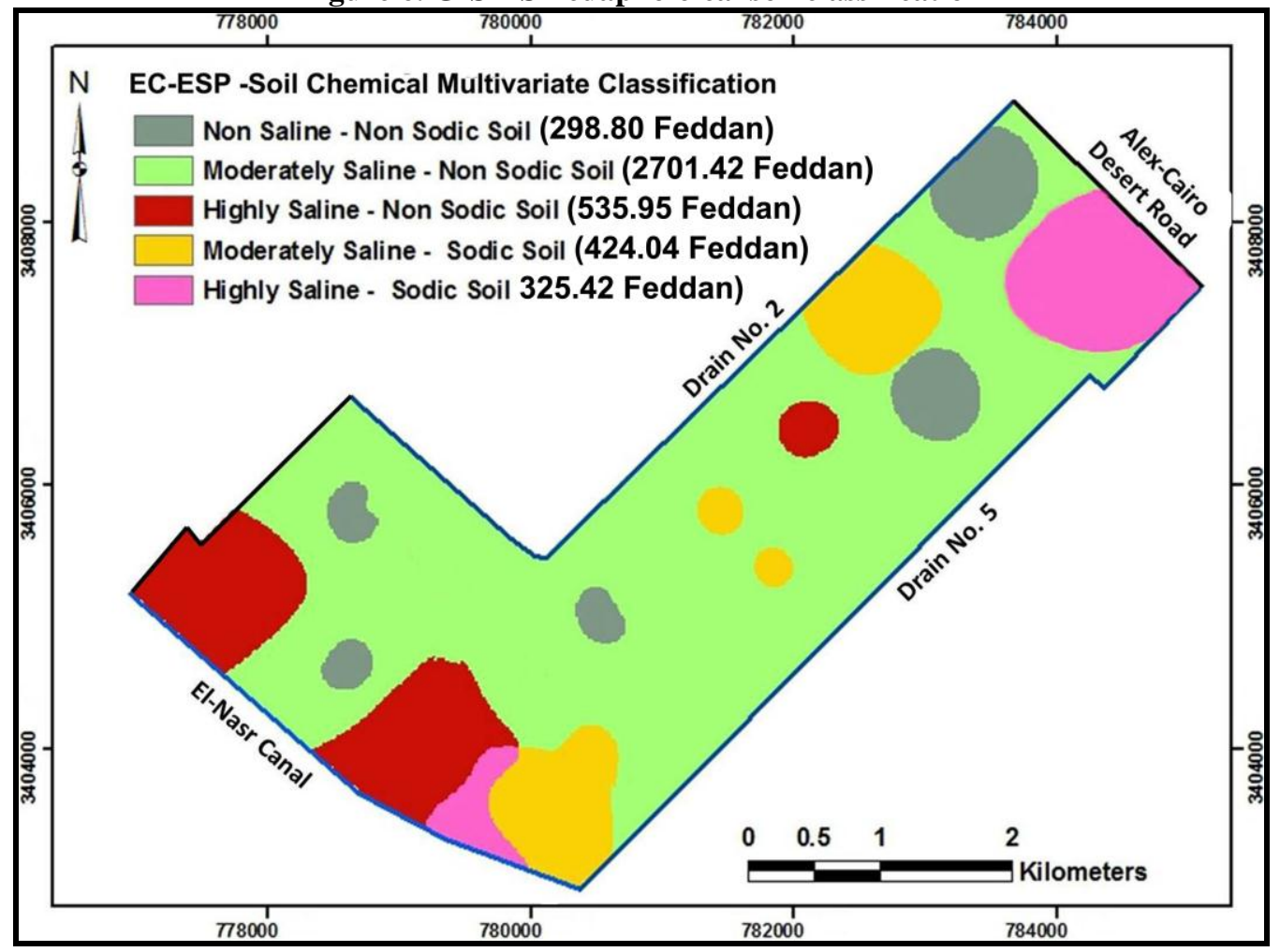

Figure 7. EC-ESP soil variants soil chemical classification overlaid map 
Table 16. Agriculture soil suitability for each profile for the selected crops in the study area

\begin{tabular}{|c|c|c|c|c|c|c|}
\hline \multirow{2}{*}{$\begin{array}{c}\text { Profile } \\
\text { No. }\end{array}$} & \multicolumn{6}{|c|}{ Crops } \\
\hline & Wheat & Faba_bean & Grape & Olive & Potato & Tomato \\
\hline 1 & $\mathrm{~S} 4(\mathrm{t}, \mathrm{hr})$ & S4 (ece, t, hr) & S2 (ece) & S2 & S2 (ece) & S1 \\
\hline 2 & $\mathrm{~S} 4(\mathrm{t}, \mathrm{hr})$ & $\mathrm{S} 4(\mathrm{ece}, \mathrm{t})$ & S3 (ece, hr) & $\mathrm{S} 3(\mathrm{dp}, \mathrm{hr})$ & $\mathrm{S} 3$ (ece, hr) & S2 (ece, hr) \\
\hline 3 & $\mathrm{~S} 3(\mathrm{t}, \mathrm{hr})$ & $\mathrm{S} 2(\mathrm{t})$ & $\mathrm{S} 2$ & S1 & $\mathrm{S} 2$ & S1 \\
\hline 4 & $\mathrm{~S} 3(\mathrm{t}, \mathrm{hr})$ & S2 & $\mathrm{S} 2$ & S2 (dp) & S2 & S1 \\
\hline 5 & $\mathrm{~S} 3(\mathrm{t}, \mathrm{hr})$ & S3 (ece, hr) & $\mathrm{S} 2$ & $\mathrm{~S} 2$ & $\mathrm{~S} 2$ & $\mathrm{~S} 2$ \\
\hline 6 & $\mathrm{~S} 4(\mathrm{t}, \mathrm{hr})$ & $\mathrm{S} 4$ (ece, $\mathrm{t}, \mathrm{esp}, \mathrm{hr}$ ) & S3 (ece, esp) & $\mathrm{S} 2$ (esp) & S4 (ece, esp) & S2 (ece) \\
\hline 7 & $\mathrm{~S} 4(\mathrm{t}, \mathrm{hr})$ & $\mathrm{S} 3$ (ece, $\mathrm{t}, \mathrm{esp})$ & S3 (esp) & S3 (dp, esp) & S3 (ece, esp) & $\mathrm{S} 2$ \\
\hline 8 & $\mathrm{~S} 3(\mathrm{t}, \mathrm{hr})$ & $\mathrm{S} 2(\mathrm{t})$ & $\mathrm{S} 2$ & $\mathrm{~S} 2(\mathrm{dp})$ & $\mathrm{S} 2$ & S1 \\
\hline 9 & $\mathrm{~S} 4(\mathrm{t}, \mathrm{hr})$ & $\mathrm{S} 4$ (ece, $\mathrm{t}, \mathrm{esp})$ & $\mathrm{S} 3$ (ece, dp, esp) & NS2 & S3 (ece, esp) & S2 (ece) \\
\hline 10 & S4 (ece, t, hr) & $\mathrm{S} 4(\mathrm{ece}, \mathrm{t})$ & $\mathrm{S} 4(\mathrm{ece}, \mathrm{dp})$ & $\mathrm{NS} 2$ & $\mathrm{~S} 4$ (ece) & S3 (ece) \\
\hline 11 & $\mathrm{~S} 3(\mathrm{t}, \mathrm{hr})$ & S3 (ece, t, hr) & S2 & $\mathrm{S} 2$ & $\mathrm{~S} 2$ & S2 \\
\hline 12 & $\mathrm{~S} 4(\mathrm{t}, \mathrm{hr})$ & S4 (ece, t, hr) & $\mathrm{S} 2($ ece, $\mathrm{t})$ & $\mathrm{S} 2(\mathrm{t})$ & S3 (ece, $t)$ & $\mathrm{S} 2($ ece, $\mathrm{t})$ \\
\hline 13 & $\mathrm{~S} 3(\mathrm{t}, \mathrm{hr})$ & $\mathrm{S} 3$ (ece, esp) & $\mathrm{S} 2$ (esp) & S2 (dp, esp) & $\mathrm{S} 2$ (esp) & $\mathrm{S} 1$ \\
\hline 14 & $\mathrm{~S} 4(\mathrm{t}, \mathrm{hr})$ & $\mathrm{S} 3(\mathrm{t})$ & $\mathrm{S} 2(\mathrm{t})$ & $\mathrm{S} 3(\mathrm{dp}, \mathrm{t})$ & $\mathrm{S} 2(\mathrm{t})$ & $\mathrm{S} 2(\mathrm{t})$ \\
\hline 15 & $\mathrm{~S} 4(\mathrm{t}, \mathrm{hr})$ & $\mathrm{S} 3(\mathrm{t}, \mathrm{hr})$ & $\mathrm{S} 2(\mathrm{t})$ & $\mathrm{S} 2(\mathrm{t})$ & $\mathrm{S} 2(\mathrm{t})$ & $\mathrm{S} 2(\mathrm{t})$ \\
\hline 16 & $\mathrm{~S} 3(\mathrm{t}, \mathrm{hr})$ & S3 (ece, $t)$ & S2 & S2 & $\mathrm{S} 2$ & S1 \\
\hline 17 & $\mathrm{~S} 4(\mathrm{t}, \mathrm{hr})$ & $\mathrm{S} 3$ (ece, $\mathrm{t}, \mathrm{hr})$ & $\mathrm{S} 2$ & $\mathrm{~S} 1$ & S2 (ece) & S2 \\
\hline 18 & $\mathrm{~S} 3(\mathrm{t}, \mathrm{hr})$ & $\mathrm{S} 2(\mathrm{ece}, \mathrm{t})$ & S2 (hr) & $\mathrm{S} 2(\mathrm{dp}, \mathrm{hr})$ & S2 (hr) & S2 (hr) \\
\hline 19 & $\mathrm{~S} 3(\mathrm{t}, \mathrm{hr})$ & $\mathrm{S} 3($ ece, $\mathrm{t})$ & $\mathrm{S} 2$ & S2 & $\mathrm{S} 2$ & S1 \\
\hline 20 & $\mathrm{~S} 3(\mathrm{t}, \mathrm{hr})$ & S3 (ece, hr) & $\mathrm{S} 2$ & $\mathrm{~S} 2(\mathrm{dp})$ & S2 & S1 \\
\hline 21 & $\mathrm{~S} 4(\mathrm{t}, \mathrm{hr})$ & $\mathrm{S} 4(\mathrm{ece}, \mathrm{t})$ & S3 $($ ece, $t)$ & $\mathrm{S} 3(\mathrm{dp}, \mathrm{t})$ & S4 (ece, $t)$ & $\mathrm{S} 2(\mathrm{ece}, \mathrm{t})$ \\
\hline 22 & $\mathrm{~S} 3(\mathrm{t}, \mathrm{hr})$ & $\mathrm{S} 2(\mathrm{t})$ & $\mathrm{S} 1$ & $\mathrm{~S} 2(\mathrm{dp})$ & $\mathrm{S} 1$ & $\mathrm{~S} 1$ \\
\hline 23 & $\mathrm{~S} 4(\mathrm{t}, \mathrm{hr})$ & $\mathrm{S} 3$ (ece, $\mathrm{t}, \mathrm{esp}, \mathrm{hr}$ ) & $\mathrm{S} 2$ (esp) & $\mathrm{S} 2$ (dp, esp) & S2 (esp) & S1 \\
\hline 24 & $\mathrm{~S} 4(\mathrm{t}, \mathrm{hr})$ & S4 (ece, t, esp, hr) & $\mathrm{S} 3$ (ece, dp, t, esp) & NS2 & $\mathrm{S} 3$ (ece, t, esp) & $\mathrm{S} 3($ ece, $\mathrm{t})$ \\
\hline 25 & $\mathrm{~S} 4(\mathrm{t}, \mathrm{hr})$ & $\mathrm{S} 3(\mathrm{t}, \mathrm{hr})$ & $\mathrm{S} 2(\mathrm{t})$ & $\mathrm{S} 2(\mathrm{dp}, \mathrm{t})$ & $\mathrm{S} 2(\mathrm{t})$ & $\mathrm{S} 2(\mathrm{t})$ \\
\hline
\end{tabular}

The limitation factors: Soil Electrical Conductivity (ece), Exchangeable Sodium Percentage (esp), Texture (t), Hydraulic Conductivity (hr), Soil Depth (dp).

Table 17. Soil suitability classes for different crops

\begin{tabular}{|c|c|c|c|c|c|c|c|c|c|c|c|c|}
\hline \multirow{2}{*}{$\begin{array}{c}\text { Suitability } \\
\text { Classes }\end{array}$} & \multicolumn{2}{|c|}{ Wheat } & \multicolumn{2}{|c|}{ Feba bean } & \multicolumn{2}{|c|}{ Grape } & \multicolumn{2}{|c|}{ Olive } & \multicolumn{2}{|c|}{ Potato } & \multicolumn{2}{|c|}{ Tomato } \\
\hline & Feddan & $\%$ & Feddan & $\%$ & Feddan & $\%$ & Feddan & $\%$ & Feddan & $\%$ & Feddan & $\%$ \\
\hline $\mathrm{S} 1$ & $\ldots \ldots$ & $\ldots \ldots$ & $\ldots \ldots$ & $\ldots \ldots$ & 295.2 & 6.9 & 234.8 & 5.5 & 295.2 & 6.9 & 1734.5 & 40.5 \\
\hline $\mathrm{S} 2$ & $\ldots \ldots$ & $\ldots .$. & 929.8 & 21.7 & 2735.0 & 63.8 & 2763.8 & 64.5 & 2648.3 & 61.8 & 2037.6 & 47.5 \\
\hline $\mathrm{S} 3$ & 1830.9 & 42.7 & 2042.1 & 47.6 & 1117.6 & 26.1 & 696.9 & 16.3 & 841.7 & 19.6 & 513.6 & 12.0 \\
\hline $\mathrm{S} 4$ & 2454.8 & 57.3 & 1313.8 & 30.7 & 137.8 & 3.2 & $\ldots .$. & $\ldots .$. & 500.5 & 11.7 & $\ldots$. & $\ldots .$. \\
\hline NS1 & $\ldots \ldots$ & $\ldots \ldots$ & $\ldots \ldots$ & $\ldots \ldots$ & $\ldots \ldots$ & $\ldots \ldots$ & $\ldots \ldots$ & $\ldots$. & $\ldots \ldots$ & $\cdots \cdots$ & $\ldots \ldots$ & $\ldots \ldots$ \\
\hline NS2 & $\ldots \ldots$ & $\ldots \ldots$ & $\ldots \ldots$ & $\ldots \ldots$ & $\ldots \ldots$ & $\ldots \ldots$ & 590.2 & 13.8 & $\ldots \ldots$ & $\ldots \ldots$ & $\ldots \ldots$ & $\ldots \ldots$ \\
\hline
\end{tabular}




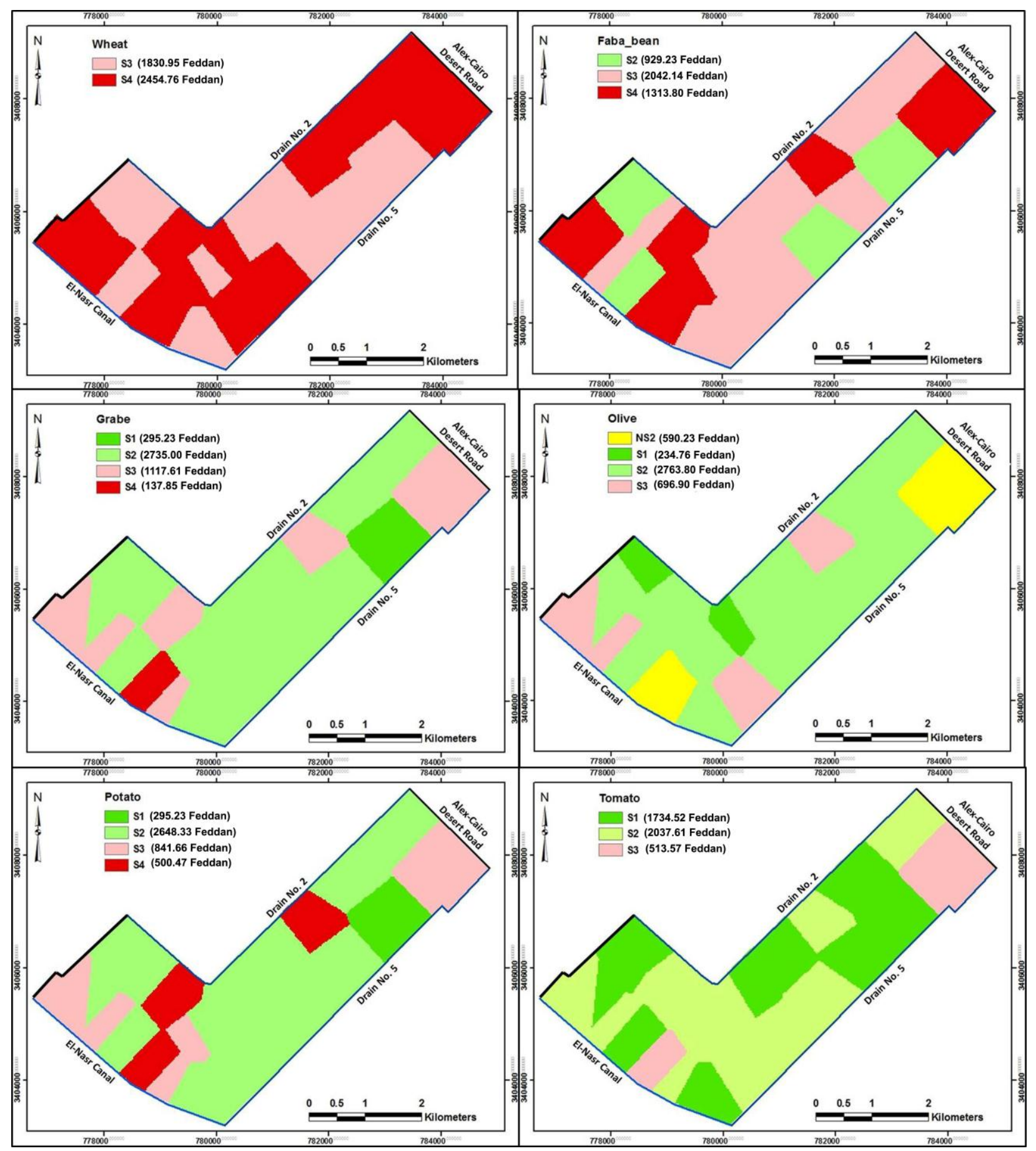

Figure 8. Soil suitability classes for selected crops

- Vegetables (Exa. Potato and Tomato): The results of this study showed that all area is suitable for potato plantation; $61.8 \%$ of the study area is moderately suitable (S2), $19.6 \%$ is marginally suitable (S3), $11.7 \%$ is conditionally suitable (S4) and $6.9 \%$ is highly suitable (S1) (Table 17 and Figure 8). This high potato soil suitability may explained as potatoes ability to be adapted to a wide range of soil types, though a deep, well-drained sandy loam is ideal (Nunn and Qian, 2010).

Generally, Tomato grows best under temperature of 20-27 ${ }^{\circ} \mathrm{C}$. fruit setting is poor when average temperature exceed $30^{\circ} \mathrm{C}$ or fall below $10^{\circ} \mathrm{C}$. it prefer a well-drained soil because they are sensitive to water logging, and grows on all types of soil, but is best adapted in light, 
well drained and fertile soils with a neutral to slightly acid pH of 5.5 to 7.0. (Bagli et al., 2003). The results of this study showed that all soil is suitable for tomato plantation (Table 17 and Figure 8). The largest portion of the study area $(88.0 \%)$ was classified as highly suitable (S1) and moderately suitable (S2). The remaining portion $(12.0 \%)$ was classified as marginally suitable (S3).

\section{CONCLUSIONS}

Physical and chemical soil characterization led to soil numerical classification that guide accurately and quantitatively the practices of soil management and reclamation. Comprehensive analytical approach of soil evaluation may be based on the processes of soil numerical classification, land suitability and crop soil suitability. GIS-EC edapholical maps are trustworthy tools to quantify the spatial distribution of leaching requirements for saline soils and gypsum requirements for reclaiming sodic soils. GIS-EC and ESP overlaid maps conduct to output the soil multivariable chemical classification. The leaching requirements (LR) equation of Bauder et al., 2018, is not applicable when min ECei (initial EC soil) is lower than ECef (EC reference crop) because it outputs false values indicating the LR needs. As well as, no need for gypsum application when RNaex $\geq$ Soil initial Naex .

Hence land suitability analysis outputs reliable information related cropping and limitation opportunity and limitation, it provides great contribution for reliable and economic land use. Soil suitability information could be used by farmers to select suitable crops for their soil, as well as an accurate database and guide maps for decision makers. Crops soil suitability is mainly determined by soil properties, crop rooting depth, and EC and ESP crops tolerance. After reducing or elimination of soil limiting factors the studied area may be cultivated wheat, faba bean, fruits (grape and olive) and vegetables (potato and tomato). Some selected crops such as grape, olive, potato and tomato are recommended to be grown in the study area .Most of marginally suitable land with severe limiting factors such as soil depth, texture, EC, Ks and ESP hazards located in the northern east higher part of the study area.

\section{REFERENCES}

Abd El-Aziz, S.H. 2018. Soil capability and suitability assessment of Tushka area, Egypt by using different programs (ASLE, Microleis and modified STORIE index). Malaysian Journal of Sustainable Agriculture (MJSA) 2(2): $\quad 09-15 . \quad$ DOI: http://doi.org/10.26480/mjsa.02.2018.09.15.

Abd El-Hady A.M. 2009. Soil reclamation, p. 257, Dahmanhour University, Egypt.
Abdel Kawy, O.R. 2004. Integrating GIS, remote sensing and modeling for agricultural land suitability evaluation at east Wadi el-Natrun, Egypt. M. SC. Thesis, Faculty of Agriculture, University of Alexandria, Egypt.

Abdelaty, E.F. 2015. GIS-mapping aridity and rainfall water deficit of Egypt. J. Agric. \& Env. Sci. Dam. Univ., Egypt. Vol.14 (2):17-40.

Abdelaty, E.F. 2016. Land use change detection and prediction using high-spatial resolution Google earth imagery and GIS techniques: A study on ElBeheira Governorate, Egypt. Fourth International Conference on Remote Sensing and Geoinformation of the Environment (RSCy2016)، Proc. of SPIE : 9688, 968803. SPIE - CCC code: $0277-786 \mathrm{X} / 16 / \$ 18$. doi: 10.1117/12.2239898.

Abdelaty, E.F. 2018. Monitoring of water quality for agriculture purposes using high resolution images (ASTER): A case study from Egypt. Alex. Sci. Exch. J. Vol. 39. No.3:465-477.

Abdel-Hamid, M.A., M.Ismail Y.A. Nasr and Y. Kotb.2010. Assessment of soils of Wadi El-Natrun area, Egypt using remote sensing and GIS techniques, Journal of American science 2010. 6 (10).

Abd-ElMabod, S.K., A. Jordán, M. Anaya-Romero, R.R. Ali, M.Muñoz-Rojas, L.M. Zavala and D. de la Rosa. 2012. Impact of topography and soil factors on crop suitability in two Mediterranean areas (Egypt and Spain) Geophysical Research Abstracts. 14.EGU2012-443.

Afify, A.A., M.E. Wahdan and F.M. Fahmy.2016. Characterizing land cover, physiography and soils in a representative area of different soil parent materials East of River Nile in El Menia governorate, Egypt. J. Soil Sci. and Agric. Eng. Mansoura Univ. Vol. 7 (7): 469 - 476.

Aldabaa, A.A.A. and E.A.M. Khralifa.2016. Evaluation of Wadi Hudein Delta's soils, Al-Shalatien, Southeastern Egypt. Alex. J. Agric. Sci. 61. No.4: 383-398.

Aldabaa, A.A., H. Zhang, A. Shata, S. El-Sawey, A. AbdelHameed and J.L. Schroder. 2010. Land suitability classification of a desert area in Egypt for some crops using Microleis program. American-Eurasian J. Agric. \& Environ. Sci. 8 (1): 80-94: 1818-6769.

ArcMap 10.2. 2008. ESRI, Environmental Systems Research Institute. U.S. copyright 2008 ESRI Inc.

Bagli, S., J.M. Terres, J. Gallego, A. Annoni and J.F. Dallemand. 2003. Agro-pedo-climatological zoning of Italy, definition of homogeneous suitable agropedoclimatic zones, application to grain maize, durum wheat, soft wheat, spring barley, sugar beet, rapeseed, sunflower, soybean, tomato.European commission, monograph 20550 En, E.C. Italy.

Bauder, T.A., J.G. Davis and R.M. Waskom.2018. Managing saline soils, fact sheet No. 0.503 pp: 1-5, Extension Colorado State University, U.S. http://extension.colostate.edu/topicareas/agriculture/managing-saline-soils-0-503 
Bucelli, P. and E. Costantini. 2009. Manual of methods for soil and land evaluation, Grape and Vine Zoning.Florence. pp.387

Canada Government 1998. Canadian system of soil classification, 3rd edition: Chapter 15: Soil Phase Agriculture and Agri-Food Canada http://sis.agr.gc.ca/cansis/taxa/cssc3/chpt15.html

Diaz, D.R. and D. Presley.2017. Management of saline and sodic soils. K-State research and extension, Kansas State University, MF1022. https://www.bookstore.ksre.ksu.edu/pubs/mf1022.pdf

Division of agriculture and natural resources, University of California. 2018. Crop rooting depth - UC drought management, Source: Chapter 11, "Sprinkler Irrigation," Section 15, natural resources conservation service national engineering handbook http://ucmanagedrought.ucdavis.edu/Agriculture/Irrigation _Scheduling/Evapotranspiration_Scheduling_ET/Frequen cy_of_Irrigation/Crop_Rooting_Depth

Duiker, S.W. 2002. Diagnosing soil compaction using a penetrometer (soil compaction tester) Penn state extension. https://extension.psu.edu/diagnosing-soilcompaction-using-a-penetrometer-soil-compaction-tester Bottom of Form

El Baroudy, A.A. 2016. Mapping and evaluating land suitability using a GIS-based model. Catena 140: 96-104. 0341-8162.

ElGhonamey, Y.K. 2015. Evaluation of some soils in north western Paris oasis (Egypt) using STORIE index and SYS models. Egypt. J. Agric. Res. 93 (1)

Elnaggar, A.A. 2017. Development of land capability and suitability maps for Bahariya Oasis, Egypt. Egypt. J. Soil Sci. Vol. 57. No. 4. pp:489 - 503

Fadl, M.E. and Abuzaid, A.S. 2017. Assessment of land suitability and water requirements for different crops in Dahrla Oasis, Western desert, Egypt. International Journal of Plant \& Soil Science. 16(6): 1-16.

FAO.1970. Physical and chemical methods of soil and water analysis, soils bull. No. 10, food and agriculture organization, Rome, Italy

FAO.1979. Land evaluation criteria for irrigation, soils bulletin No. 50, FAO, Rome

FAO. 2018a. Evaluation of FAO's contribution to the Arab Republic of Egypt 2012-2017

FAO. 2018b. FAO soils portal. http://www.fao.org/soilsportal/soil-survey/soil-classification/numerical-systems/

Franchini, E. 2009. Manual of methods for soil and land evaluation, olive tree (Olea europea L.,Florence, pp:401420.

George A.P. 1960. Tolerance of crops to exchangeable sodium, agricultural information bulletin no.216. pp: 1-4, agriculture research service, soil and water conservation research division, agricultural research service. U.S dept. of agriculture.
Gurung, T.R. and A.K. Azad.2013. Best practices and procedures of saline soil reclamation systems in SAARC countries. SAARC agriculture center, ISBN: 978-984-336355-8 http://www.sac.org.bd/archives/publications/Saline\%20So il\%20Reclamation.pdf

Ismail, H.A., E.M. El-Zahaby and M.E. El-Fayoumy.1994. A modified approach for land evaluation under arid condition. 11. Applications. J. Agric. Set., Mansoura Univ. 19 (10). 3497

Ismail, H.A., I. Morsy, El-Zahaby, E.M. and El-Nagar, F.S. 2001. A developed expert system for land use planning by coupling land information system and modeling. Alex. J. Agric. Res. 46 (3). 141.

Joerin, F., M. Thériault, and A. Musy.2001. Using GIS and outranking multicriteria analysis for land-use suitability assessment, International Journal of Geographical Information Science. 15(2): 153-174. 1365- 8816 print/ISSN1362-3087onlineTaylor \& Francis Ltd doi: 10.1080/13658810051030487

Kassim, Y., M. Mahmoud, S. Kurdi, and C. Breisinger. 2018. An agricultural policy review of Egypt, first steps towards a new strategy. IFPRI-Middle East and North Africa, Regional program, working paper 11

Kupper, K.H. 1996. Improved Irrigation and Drainage of Saline Soils, Inst. Drainage and Irrigation, Netherland

Lanyon, D.M., A. Cass and D. Hansen.2004. The effect of soil properties on vine performance. CSIRO Land and Water Technical Report No. 34/04. 1446-6171

Lenntech, 2018. SAR hazard of irrigation water: Sodium hazard of irrigation water https://www.lenntech.com/applications/irrigation/sar/sarhazard-of-irrigation-water.htm

Mishra, S.K. 2004. Median as a weighted arithmetic mean of all sample observations. Economics Bulletin, Vol. 3. 18: $1-6$

Mohammed, A. 2006. Land use/cover dynamics over a period of three decades

in the Jello micro-catchment, Chercher highlands, Ethiopia. Ethiopian Journal of Natural Resources: 1563-3705

Nunn, N. and N.Qian, 2010. The Columbian Exchange: A History of Disease, Food, and Ideas. Journal of Economic Perspectives. (24). (2): 163-188.

Page, A.L., R.H. Miller and D.R. Keeney.1982. Methods of Soil Analysis: 2- Chemical and Microbiological Properties, 2. Aufl., 1184 S., American Soc. of Agronomy (Publ.), Madison, Wisconsin, USA, gebunden 36 Dollar.

Pan, G. and J. Pan. 2012. Research in crop land suitability analysis based on GIS, Computer and Computing Technologies in Agriculture. 365: 314-325.

Prapagar, K., S.P. Indraratne and P. Premanandharajah. 2012. Effect of Soil Amendments on Reclamation of SalineSodic soil , Tropical agricultural research . 23 (2): 168 176 
Qadir, M., A. Tubeileh, J. Ahrtar, A. larbi, P.S. Minhas and M.A. Hran. 2008. Productivity Enhancement of SaltAffected Environments Through Crop Diversification. Land Degrad. Develop. 19: 429-453. DOI: 10.1002/ldr.853

www.halophyte.org/pdfs/drhran_pdfs/152.pdf

Singha, C. and K.C. Swain. 2016. Land suitability evaluation criteria for agricultural crop selection: A review. Agricultural reviews. 37. (2):125-132 Print ISSN: $0253-$ 1496 / Online ISSN: 0976-0539

Stibinger, J. 2014. Examples of determining the hydraulic conductivity of soils, theory and applications of selected basic methods, University handbook on soil hydraulics,
Jan Evangelista Purkyně University, Faculty of the Environment. ISBN 978-80-7414-836-1.

Viji, R. and P.P. Rajesh. 2012. Assessment of water holding capacity of major soil series of Lalgudi, Trichy, India. J. Environ. Res. Develop. Vol. 7 No. 1A.

Wafaa, H.M. 2013. A yield quality and micronutrients uptake of carrot (Daucus carota L.) and some soil properties as affected by organic fertilizers and elemental sulphur application. Egypt. J. Soil Sci. 53. 4. Pp: 537-554

Wahab, M.A., M.A. El Semary, R.R. Ali, and Hr.M. Darwish. 2013. Land resources assessment for agricultural use in some areas west of Nile valley, Egypt. Journal of Applied Sciences Research. 9 (7): 4288-4298, ISSN 1819-544X. 


\section{الملخص العربي}

\section{النهج التحليلي الشامل لنظم المعلومات الجغرافية لاستخدامات الأراضي عن طريق ريط صلاحية التربة

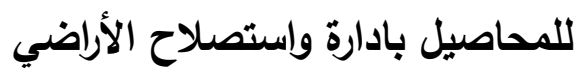

$$
\text { عبد رب النبي محمد عبد الهادي و عماد فوزي عبد العاطي }
$$

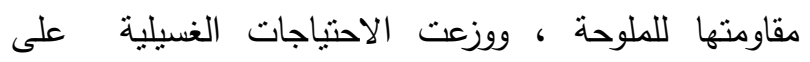
خريطة ملوحة التربة والتى تكونت من أربعة وحدات خرائطية

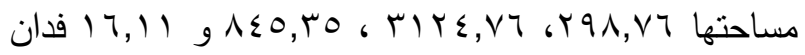
، وتميزت الخريطة كذلك بتحديد التوزيع المكاني للاحتياجات الغسيلية ، وذللك على النحو التالى (أ) الاحتياجات الغسيلية

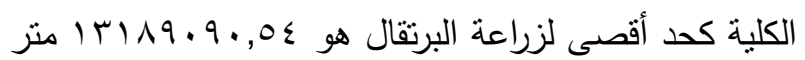
مكعب لكل منطقة الدراسة. (ب) القمح أكثر تحملا للملوحة من البرتقال لذا أنخفضت الاحتياجات الغسيلية الاجمالية فى

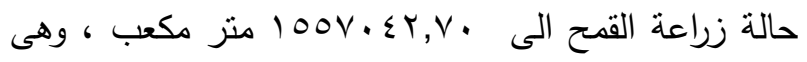
أقل بكثير منها في حالة زراعة البرتقال.

رسمت الخريطة الايدافولوجية لملوحة وصودية التربة

GIS-ESP ارتباطا بمقاومة المحاصيل لملوحة التربة (edapholoical soil classification ) ، والتى قسمت منطقة three categories of الدراسة إلى ثلاث مراتب من الأراضي ESP tolerance crop soil الحساسية للصوديوم (9., (1) ف فدان) - وحدة خرائطية

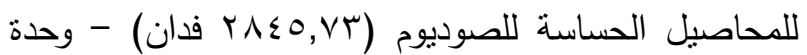
خرائطية للمحاصيل المتحملة للصوديوم ( وأستخدمت هذه الخربطه في حساب وتحديد التوزيع المكانى للاحتياجات الجبسية وفقا للمحاصيل المختلفة. رسمت خريطة متعددة المتغيرات تجمع خريطة الملوحه GIS-EC and ESP overlaid مع خريطة الصوديوم المنبادل maps ، وتتكونت هذه الخريطة من خمس مراتب : نربة غير

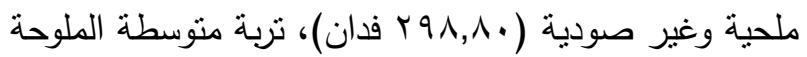

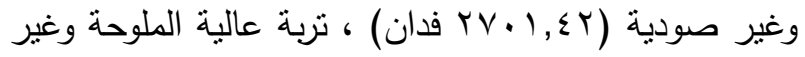
صودية (0,90به فدان)، تربة متوسطة الملوحة وصودية
تمتلك منطقة طريق القاهرة- الأسكندرية الصحراوي (مصر) مقدرات زراعية للمساهمة في الأمن الغذائي القومي

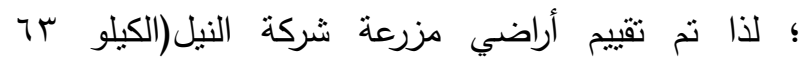
باالصحراوي) ، بتطبيق المنهج التحليلي الشامل، حيث أجرى لهى تقسيم رقمى للأراضى بناء على خصائصها الفيزيائية والكيميائية، الأمر الذى ساعد على تحديد مدى ملائمة الأراضى للمحاصيل وتوجيه ممارسات إدارة وأستصلاح

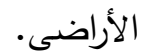

أشنار التقسيم الرقمى (أحادي المتغير) الفيزيائي univariate numerical soil classification الرئيسية ئأراضي منطقة الدراسة والتى رسمت خرائطها بأستخدام نظم المعلومات الجغرافية GIS، حيث أوضحت الخرائط مساحات الأنماط الرئيسية : نربة متوسطة العمق (1997,V7 (فدان) - نربة ذات نفاذية متوسطة

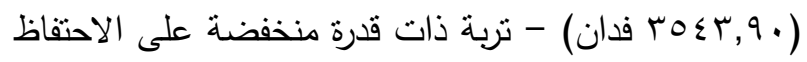

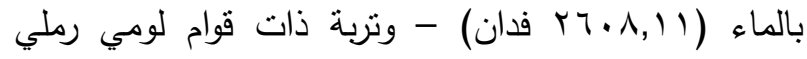

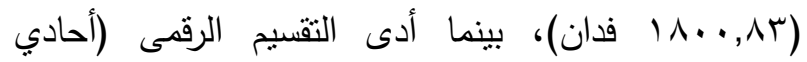
soil المتغير) الكيميائي إلى بيان مساحة الصفوفئ

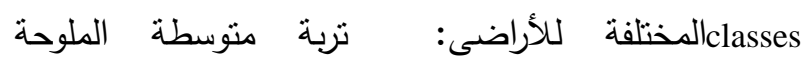

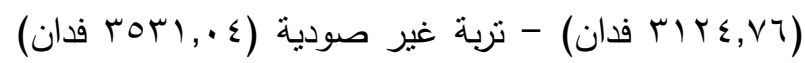
- وتربة غير كلسية (0r, (01 ب فدان). كنوع من الممارسة الجيدة cultural practice لإدارة ملوحة التربة، فقد أثشارت الدراسة إلى توليفة المحاصيل combination التي تتحمل درجات مختلفة من of EC- tolerance crops ملوحة التربة، وحسبت الاحتياجات الغسيلية لأغراض أستصلاح الأراضى الملحية لزراعة محاصيل مختلفة في 
S3 = ) ، ك2 =63.8\% ( أما صلاحيتها للعنب فبلغت 26.1\%). يعتبر معظم المنطقة صالحة لزراعة الزينون

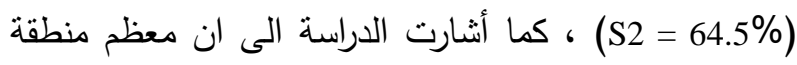
S2 = (الدراسة كانت صالحة لزراعة محصول البطاطس S1 = (61.79\%) ، (S3 = 19.64\%) وكذلك لمحصول الطماطح ) (S3)

$$
.(\mathrm{S} 2=47.5 \%) \cdot(40.5 \%)
$$

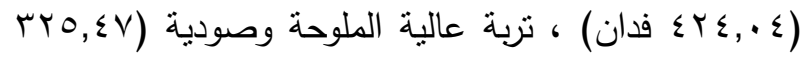
فدان). حددت المعوقات الرئيسية main limiting factors للتربة بخريطة ملائمة الاراضي لزراعة المحاصيل والتى أوضحت أن صلاحية التربة لزراعة محصول القمح (S4 = 57.3\%) و (S3 = 42.7\%). بالنسبة لزراعة الفول ، كانت الصلاحية

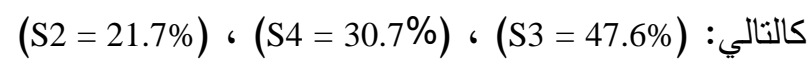

